\title{
Dipole Moment Calculations Using \\ Multiconfiguration Pair-Density Functional Theory and Hybrid Multiconfiguration Pair-Density Functional Theory
}

\author{
Aleksandr O. Lykhin ${ }^{1}$, Donald G. Truhlar,,$^{2 *}$ and Laura Gagliardi ${ }^{1,3^{*}}$ \\ ${ }^{1}$ Department of Chemistry, Pritzker School of Molecular Engineering, The James Franck Institute and Chicago \\ Center for Theoretical Chemistry, The University of Chicago, Chicago, IL 60637, United States \\ ${ }^{2}$ Department of Chemistry, Chemical Theory Center, and Minnesota Supercomputing Institute, University of \\ Minnesota, Minneapolis, MN 55455, United States \\ ${ }^{3}$ Argonne National Laboratory, Lemont, Illinois 60439, United States
}

\begin{abstract}
The dipole moment is the molecular property that most directly indicates molecular polarity. The accuracy of computed dipole moments depends strongly on the quality of the calculated electron density, and the breakdown of single-reference methods for strongly correlated systems can lead to poor predictions of the dipole moments in those cases. Here, we derive the analytical expression for obtaining the electric dipole moment by multiconfiguration pair density functional theory (MC-PDFT), and we assess the accuracy of MC-PDFT for predicting dipole moments at equilibrium and nonequilibrium geometries. We show that MC-PDFT dipole moment curves have reasonable behavior even for stretched geometries, and they significantly improve upon the CASSCF results by capturing more electron correlation. The analysis of a dataset consisting of 18 first-row transition metal diatomics and 6 main-group polyatomic molecules with multireference character suggests that MC-PDFT and its hybrid extension (HMC-PDFT) perform comparably to CASPT2 and MRCISD+Q methods and have a mean unsigned deviation of $0.2-0.3 \mathrm{D}$ with respect to the best available dipole moment reference values. We explored the dependence of the predicted dipole moments upon the choice of the on-top density functional and active space, and we recommend the tPBE and hybrid tPBE0 on-top choices for the functionals combined with the moderate correlated participating orbital scheme for selecting the active space. With these choices, the mean unsigned deviations (in debyes) of the calculated equilibrium dipole moments from the best estimates are 0.77 for CASSCF, 0.29 for MC-PDFT, 0.24 for HMC-PDFT, 0.28 for CASPT2, and 0.25 for MRCISD+Q. These results are encouraging because the computational cost of MC-PDFT or HMC-PDFT is largely reduced compared to the CASPT2 and MRCISD+Q methods.
\end{abstract}




\section{INTRODUCTION}

The accurate prediction of molecular properties is one of the primary goals of computational chemistry. Experimentally, the magnitude of the dipole moment can be obtained by using microwave Stark spectroscopy, and its direction can be determined through the isotopic dependence of the rotational magnetic moment. ${ }^{1-3}$ The permanent dipole moment measures the polarity of a single molecule, and in the condensed phase it is one of the properties that has a large effect on solvation energies. The dipoledipole interactions are important contributors to intermolecular forces, such as the ones that facilitate protein folding, self-assembly of $\pi$-conjugated dyes, and liquid crystal alignment ${ }^{4-6}$. The interaction between the permanent electric dipole moment and an external electric field can be used to manipulate the reactivity of ultracold diatomic molecules. ${ }^{7,8}$ Furthermore, dipole moments are useful molecular descriptors $;^{9,10}$ for example, in machine learning, the partitioning of a continuous molecular charge distribution into the atom-centered point charges is often restrained to reproduce the dipole moments (or other physical observables). ${ }^{11,12}$ The partial atomic charges can then be used in the parametrization of classical force fields, ${ }^{11}$ calculation of solvation free energies, ${ }^{13}$ and evaluation of many other molecular properties. $^{14}$

The dipole moments can be used to estimate the intensity of infrared (IR) transitions. ${ }^{21}$ Dipole moments at nonequilibrium geometries are used to predict the radiative lifetimes of excited vibrational states, ${ }^{17}$ and dipole moment surfaces (DMSs) computed on a grid of geometries are used to determine intensities of transitions in rovibrational spectra. ${ }^{18-22}$ In the case of flexible molecules with rich conformational space, these intensities can be estimated from the dipole moment autocorrelation functions $^{23,24}$ propagated in $a b$ initio molecular dynamics (AIMD) simulations. ${ }^{25,26}$ To extract a full vibrational spectrum from the autocorrelation function in AIMD, the permanent dipole moment needs to be evaluated as a function of geometry, including geometries characterized by stretched chemical bonds. Single-reference electronic structure methods are often inadequate for stretched bonds because they do not capture static electron correlation. Furthermore, for strongly correlated systems, the static correlation is large even at the equilibrium geometry. In addition, the dipole moment can be sensitive to dynamic correlation; therefore, in order to compute reliable DMSs, one should use multireference methods that are capable of capturing most of the dynamic electron correlation as well as the static correlation.

A multireference method designed to do this in an affordable way is multiconfiguration pairdensity functional theory (MC-PDFT), and the present contribution is concerned with using MC-PDFT to compute dipole moments. MC-PDFT is a multireference method that uses a multiconfigurational wave 
function as the reference function and computes the total energy from the kinetic energy, density, and ontop pair density of that wave function. ${ }^{27}$ While the computational cost of MC-PDFT is lower than that of multireference perturbation theories or multireference configuration interaction, it has been shown to provide good accuracy for calculating the excitation energies, bond energies, reaction barriers, and molecular geometries. ${ }^{28-33}$ In this paper, we derive an analytical procedure for computing dipole moments by MC-PDFT, we present illustrative applications, and we compare MC-PDFT dipole moments to those computed by complete active space self-consistent field (CASSCF) calculations, ${ }^{34}$ complete active space second-order perturbation theory (CASPT2), ${ }^{35}$ and multireference configuration interaction with single and double excitations and a Davidson correction for quadrupole excitations (MRCISD+Q) ${ }^{36-38}$. The applications consist of equilibrium dipole moments of 18 first-row transition metal diatomics, six polyatomic molecules, and nonequilibrium dipole moments of $\mathrm{HF}, \mathrm{CO}, \mathrm{NO}$, and $\mathrm{AlO}$ for bond-stretched geometries.

\section{MC-PDFT ANALYTICAL DIPOLE MOMENT}

In a homogeneous electric field, the energy can be expanded in a Taylor series in field strength $\mathbf{F}$ as

$$
E(\mathbf{F})=E_{0}-\boldsymbol{\mu} \cdot \mathbf{F}+\ldots
$$

so that

$$
\mu_{\eta}=-\left.\frac{\partial E}{\partial F_{\eta}}\right|_{\mathbf{F}=0} ; \eta=x, y, z
$$

where $E_{0}$ is the energy in the absence of a field, and $\boldsymbol{\mu}$ is the field-free electric dipole moment. Therefore, the electric dipole moment can be computed by differentiation of the total electronic energy with respect to the strength of the electric field. We begin by writing out the MC-PDFT energy expression in the presence of a homogeneous electric field

$$
\begin{aligned}
& E_{\mathrm{MC}-\mathrm{PDFT}}=V_{n n}+\sum_{p q} h_{p q} D_{p q}+\frac{1}{2} \sum_{p q s t} g_{p q s t} D_{p q} D_{s t}+E_{\mathrm{ot}}\left[\rho, \Pi, \rho^{\prime}, \Pi^{\prime}\right] \\
& +\sum_{p q} \sum_{\eta} F_{\eta}\left\{\mathbf{m}_{p q}\right\}_{\eta} D_{p q}-\sum_{A} \sum_{\eta} F_{\eta} Z_{A}\left\{\mathbf{R}_{A}\right\}_{\eta}
\end{aligned}
$$

where indices $p, q$, s, and $t$ refer to the molecular orbitals, $Z$ is the nuclear charge, $\mathbf{R}_{A}$ is the position vector of a nucleus, $A$ runs over all nuclei, and $\eta$ runs over $x, y$, and $z$ coordinates. The first four terms include the nuclear repulsion energy $V_{\mathrm{nn}}$, the one- and two-electron electron integrals $\left(h_{p q}\right.$ and $\left.g_{p q r s}\right)$, the elements 
of one- and two-electron reduced density matrices $\left(D_{p q}\right.$ and $\left.d_{p q r s}\right)$, and the on-top pair-density functional $E_{\text {ot, }}$ which depends on the electron density $\rho$, the on-top pair density $\Pi$, and their gradients. The last two terms account for the electric field effect. The electric dipole integrals $\mathbf{m}_{p q}$ in the $x$ direction are given by

$$
\left\{\mathbf{m}_{p q}\right\}_{X}=\left\langle\varphi_{p}\left|q_{e} x\right| \varphi_{q}\right\rangle
$$

where $\varphi$ are the basis functions, $q_{\mathrm{e}}$ is the electron charge, and $x$ is an electronic coordinate; analogous expressions hold for the $y$ and $z$ directions. Unlike a nuclear perturbation, which perturbs both one- and two-electron integrals, the electric field perturbs only the one-electron integrals. ${ }^{39}$ The present article is restricted to state-specific CASSCF, not state-averaged CASSCF or other multiconfiguration reference wave functions. We first present the theory for the original version of MC-PDFT, ${ }^{27}$ and then we present the extension to hybrid MC-PDFT (HMC-PDFT). ${ }^{40}$

$M C$-PDFT. The component of the electric dipole moment along the $x$-axis can be written as the derivative of the MC-PDFT energy with respect to the strength of the electric field $F_{x}$

$$
\mu_{X}=-\frac{\mathrm{d} E_{\mathrm{MC}-\mathrm{PDFT}}}{\mathrm{d} F_{X}}=-\left(\frac{\partial E_{\mathrm{MC}-\mathrm{PDFT}}}{\partial F_{X}}+\frac{\partial E_{\mathrm{MC}-\mathrm{PDFT}}}{\partial \mathbf{\kappa}} \cdot \frac{\partial \boldsymbol{\kappa}}{\partial F_{x}}+\frac{\partial E_{\mathrm{MC}-\mathrm{PDFT}}}{\partial \mathrm{P}} \cdot \frac{\partial \mathrm{P}}{\partial F_{X}}\right)
$$

where vectors $\boldsymbol{\kappa}$ and $\mathbf{P}$ represent, respectively, the orbital and state rotation parameters ${ }^{41,42}$ minimizing the state-specific CASSCF energy. Because MC-PDFT is a non-variational method, the $\frac{\partial \boldsymbol{\kappa}}{\partial F_{x}}$ and $\frac{\partial \mathbf{P}}{\partial F_{x}}$ partial derivatives are generally not equal to zero and thus the response of the wave function is required to compute the dipole moment. This is accomplished by using the method of Lagrange multipliers as was previously shown for the state-specific ${ }^{41}$ and state-averaged ${ }^{42,43}$ MC-PDFT analytical nuclear gradients. If the CASSCF wave function is chosen as the reference wave function in MC-PDFT, the Lagrangian takes the form

$$
L=E_{\mathrm{MC}-\mathrm{PDFT}}+\mathbf{z}^{\mathrm{orb}} \cdot \frac{\partial E_{\mathrm{CASSCF}}}{\partial \boldsymbol{\kappa}}+\mathbf{z}^{\mathrm{CI}} \cdot \frac{\partial E_{\mathrm{CASSCF}}}{\partial \mathrm{P}}
$$

where $\mathbf{z}^{\text {orb }}$ is a Lagrangian multiplier for orbital rotation, and $\mathbf{z}^{\mathrm{CI}}$ is a Lagrangian multiplier for state rotation. These multipliers are chosen to nullify the last two terms in eq. (6) and make the Lagrangian variational with respect to the $\boldsymbol{\kappa}$ and $\mathbf{P}$ vectors. Since the energies of MC-PDFT and the Lagrangian are equal, the derivative in eq (5) can be written in the form of a partial derivative of the Lagrangian with respect to the electric field: 


$$
\begin{gathered}
\frac{\mathrm{d} E_{\mathrm{MC}-\mathrm{PDFT}}}{\mathrm{d} F_{X}}=\frac{\mathrm{d} L}{\mathrm{~d} F_{X}}=\frac{\partial L}{\partial F_{X}}+\underbrace{\frac{\partial L}{\partial \mathbf{\kappa}} \cdot \frac{\partial \mathbf{\kappa}}{\partial F_{x}}}_{0}+\underbrace{\frac{\partial L}{\partial \mathbf{P}} \cdot \frac{\partial \mathbf{P}}{\partial F_{X}}}_{0}=\frac{\partial L}{\partial F_{X}} . \\
\frac{\partial L}{\partial F_{X}}=\frac{\partial E_{\mathrm{MC}-\mathrm{PDFT}}}{\partial F_{X}}+\frac{\partial}{\partial F_{X}}\left(\mathbf{z}^{\mathrm{orb}} \cdot \frac{\partial E_{\mathrm{CASSCF}}}{\partial \mathbf{\kappa}}\right)+\frac{\partial}{\partial F_{X}}\left(\mathbf{z}^{\mathrm{CI}} \cdot \frac{\partial E_{\mathrm{CASSCF}}}{\partial \mathbf{P}}\right) .
\end{gathered}
$$

Because the nuclear repulsion term, two-electron part, and on-top energy are all independent of the external electric field, the first term in eq (8) can be reduced to

$$
\begin{aligned}
\frac{\partial E_{\mathrm{MC}-\mathrm{PDFT}}}{\partial F_{x}} & =\frac{\partial}{\partial F_{x}}\left(\sum_{p q}\left[h_{p q}+\sum_{\eta} F_{\eta}\left\{\mathbf{m}_{p q}\right\}_{\eta}\right] D_{p q}-\sum_{A} \sum_{\eta} F_{\eta} Z_{A}\left\{\mathbf{R}_{A}\right\}_{\eta}\right) \\
& =\sum_{p q}\left\{\mathbf{m}_{p q}\right\}_{x} D_{p q}-\sum_{A} Z_{A}\left\{\mathbf{R}_{A}\right\}_{x}
\end{aligned}
$$

The derivatives of the second and third terms in eq (8) are reminiscent of those used in the evaluation of the MC-PDFT nuclear-coordinate gradients, ${ }^{42}$ except that the spatial derivatives of the one-electron integrals are replaced by the dipole integrals:

$$
\begin{aligned}
\frac{\partial}{\partial F_{X}}\left(\mathbf{z}^{\mathrm{orb}} \cdot \frac{\partial E_{\mathrm{CASSCF}}}{\partial \mathbf{\kappa}}\right) & =\frac{\partial}{\partial F_{X}}\left(\sum_{i j} z_{i j} \frac{\partial}{\partial \kappa_{i j}} \sum_{p q}\left[h_{p q}+\sum_{\eta} F_{\eta}\left\{\mathbf{m}_{p q}\right\}_{\eta}\right] D_{p q}\right) \\
& =\sum_{p q}\left\{\mathbf{m}_{p q}\right\}_{x} \sum_{s}\left(z_{p s} D_{s q}-z_{s q} D_{p s}\right) \\
\frac{\partial}{\partial F_{X}}\left(\mathbf{z}^{\mathrm{CI}} \cdot \frac{\partial E_{\mathrm{CASSCF}}}{\partial \mathbf{P}}\right) & =\frac{\partial}{\partial F_{X}}\left(\sum_{I} Z_{I} \frac{\partial}{\partial P_{I}} \sum_{p q}\left[h_{p q}+\sum_{\eta} F_{\eta}\left\{\mathbf{m}_{p q}\right\}_{\eta}\right] D_{p q}\right) \\
& =\sum_{p q}\left\{\mathbf{m}_{p q}\right\}_{X} \sum_{I} Z_{I}\left[\left\langle I\left|\hat{E}_{p q}\right| 0\right\rangle+\left\langle 0\left|\hat{E}_{p q}\right| I\right\rangle\right]
\end{aligned}
$$

where $\langle 0|\left\langle\hat{E}_{p q} \mid I\right\rangle$ are the elements of the transition density matrix, where $\hat{E}_{p q}$ is the spin-traced excitation operator,

$$
\hat{E}_{p q}=\hat{a}_{p \alpha}^{\dagger} \hat{a}_{q \alpha}+\hat{a}_{p \beta}^{\dagger} \hat{a}_{q \beta}
$$

and $|0\rangle$ and $|I\rangle$ are the reference and excited configuration state functions, respectively. By substituting eqs (9), (10), and (11) into eq (8) and including the minus sign of eq (5), we obtain the final expression for the state-specific MC-PDFT permanent dipole moment:

$$
\mu_{X}=-\sum_{p q}\left\{\mathbf{m}_{p q}\right\}_{X}\left(D_{p q}+\breve{D}_{p q}\right)+\sum_{A} Z_{A}\left\{\mathbf{R}_{A}\right\}_{X} \text {. }
$$


where we introduced the auxiliary matrix $\breve{\mathbf{D}}$ with elements:

$$
\breve{D}_{p q}=\sum_{s}\left(z_{p s} D_{s q}-z_{s q} D_{p s}\right)+\sum_{I} z_{I}\left[\left\langle I\left|\hat{E}_{p q}\right| 0\right\rangle+\left\langle 0\left|\hat{E}_{p q}\right| I\right\rangle\right]
$$

By rearranging terms in eq (13), one can show that that the MC-PDFT dipole moment is the sum of the CASSCF dipole moment and a correction term:

$$
\mu_{X}^{\mathrm{MC}-\mathrm{PDFT}}=\mu_{X}^{\mathrm{CASSCF}}-\sum_{p q}\left\{\mathbf{m}_{p q}\right\}_{X} \breve{D}_{p q}
$$

$H M C-P D F T$. In HMC-PDFT,$^{40}$ the energy is expressed as a weighted average of the CASSCF and MC-PDFT energy expressions:

$$
E_{\mathrm{HMC}-\mathrm{PDFT}}=\lambda E_{\mathrm{CASSCF}}+(1-\lambda) E_{\mathrm{MC}-\mathrm{PDFT}} .
$$

where $\lambda$ is a hybridization constant. Then the HMC-PDFT dipole moment is given by

$$
\mu_{x}^{\mathrm{HMC}-\mathrm{PDFT}}=\mu_{x}^{\mathrm{CASSCF}}-(1-\lambda) \sum_{p q}\left\{\mathbf{m}_{p q}\right\}_{x} \breve{D}_{p q}
$$

\section{COMPUTATIONAL METHODS}

The calculations were performed using the $P y S C F^{44,45}$ package with the $m r h^{46}$ add-on that enables pair-density functional calculations. For diatomic molecules, we used $C_{\infty v}$ symmetry, which is explicitly supported in $\mathrm{PySCF}^{47}$

We employ both MC-PDFT and HMC-PDFT. In the latter $\lambda$ is an adjustable parameter. Using $\lambda=$ 0.25 combined with tPBE functional, labeled as tPBE $0,{ }^{40}$ significantly improves the dipole moment values with no additional computational cost compared to tPBE and CASSCF, and we will thus discuss results with tPBE0 in the following. The MC-PDFT dipole moments were computed analytically as discussed in Section II using the translated on-top density functionals $\left(\mathrm{tPBE}^{27}\right.$ and $\mathrm{COPBE}^{48}$ ) and hybrid MC-PDFT $\left(\mathrm{tPBE} 0^{40}\right)$ with the grid size level set to 9 . For the fully translated ftPBE ${ }^{49}$ on-top density functional, the dipole moments were computed numerically to investigate the effect of translation scheme on the accuracy of dipole moments prior to analytical implementation.

We explored three correlated participating correlated orbital (CPO) schemes ${ }^{50-52}$ for selecting the active space for transition metal diatomics: nominal (nom-CPO), moderate (mod-CPO), and extended (ext-CPO). Each CPO active space includes multiple pairs of active molecular orbitals and active valence electrons. The first orbital in each pair is formally occupied, while the second orbital, denoted as a correlating orbital, is formally unoccupied. with the two orbitals having similar spatial distribution (see 
the natural orbitals in the SI). The nom-CPO active space includes the doubly occupied bonding orbitals, doubly occupied antibonding orbitals (if there are any), all singly occupied orbitals, and the corresponding correlating counterparts. The mod-CPO active space includes all orbitals of nom-CPO plus the $d$-subshell occupied orbitals of the metal, the $p$-subshell doubly occupied nonbonding orbitals of the non-metal (if there are any), and the correlating counterparts. Note that in the original formulation for transition metal compounds, ${ }^{51}$ the mod-CPO scheme included singly and doubly occupied $d$-subshell orbitals, whereas here we add all $d$-shell orbitals regardless of their occupation if the ground state has high symmetry, such as $\Phi$ or $\Gamma$. Finally, the ext-CPO includes all the orbitals of the mod-CPO active space plus the $4 s$ occupied orbitals of the metal, $2 s$ or $3 s$ nonbinding orbitals of the nonmetal, and the corresponding correlating orbitals.

The multireference complete active space perturbation theory (CASPT2) calculations and multireference configuration interaction calculations with single and double excitations and fixedreference Davidson correction (MRCISD+Q) ${ }^{36-38}$ (eqs 1 and 2 of ref ${ }^{53}$ ) were performed using the Molpro $^{54}$ package. In the case of CASPT2, the core orbitals were not correlated in the configuration interaction step, and an imaginary level shift of 0.2 a.u. was applied to avoid intruder state problems. In Molpro, we used $C_{2 v}$ symmetry for diatomic molecules with additional default supersymmetry constraints preventing mixing of $\delta$ and $\sigma$ orbitals in the $a_{1}$ irreducible representation and mixing of $\varphi$ and $\pi$ orbitals in $b_{1}$ and $b_{2}$ irreducible representations. The MRCISD+Q dipole moments were computed numerically using the finite-field two-point central difference formula unlike the CASPT2 dipole moments, which were computed analytically by differentiation of the energy with respect to the electric field. ${ }^{55}$ The energy convergence threshold was set to $10^{-10}$ a.u. to ensure numerical stability, and in all cases the numerical dipoles were converged within $10^{-3} \mathrm{D}$. All MRCISD calculations in this article are internally contracted.

The CASSCF energies obtained in PySCF and Molpro agreed to within $10^{-6}$ a.u., which confirms that the CASSCF solutions are identical in two programs. Since augmentation of the basis set with diffuse functions has been found to significantly improve the accuracy of dipole moments, ${ }^{56}$ the calculations at the equilibrium and nonequilibrium configurations were performed with the aug-cc-pVXZ $(X=\mathrm{T}$ or $\mathrm{Q})$ basis set. In the case of transition metal diatomics, a more compact def2-TZVPD basis set was used with diffuse functions on both hydrogen and non-hydrogen atoms.

The $M$ diagnostics ${ }^{52}$ were computed to assess the extent to which the considered molecules are multiconfigurational. 


\section{RESULTS AND DISCUSSION}

\section{A. Analytical vs Numerical Dipole Moments}

To test our dipole moment implementation in $P y S C F$, we use the asymmetric CHFClBr molecule with geometry optimized at the $\operatorname{tPBE}(2 \mathrm{e}, 2 \mathrm{o}) / \mathrm{aug}-\mathrm{cc}-\mathrm{pVDZ}$ level of theory. Here, the active space includes two active electrons in the bonding and antibonding molecular orbitals localized in the $\mathrm{C}-\mathrm{H}$ bond. The dipole moment curves shown in Figure 1 are obtained by elongating the $\mathrm{C}-\mathrm{H}$ bond from the equilibrium value of $1.1 \AA$ to $3.0 \AA$ with a step size of $0.1 \AA$ while freezing the remaining degrees of freedom. The numerical and analytical dipole moments agree at all distances within $0.001 \mathrm{D}$ (see the SI).

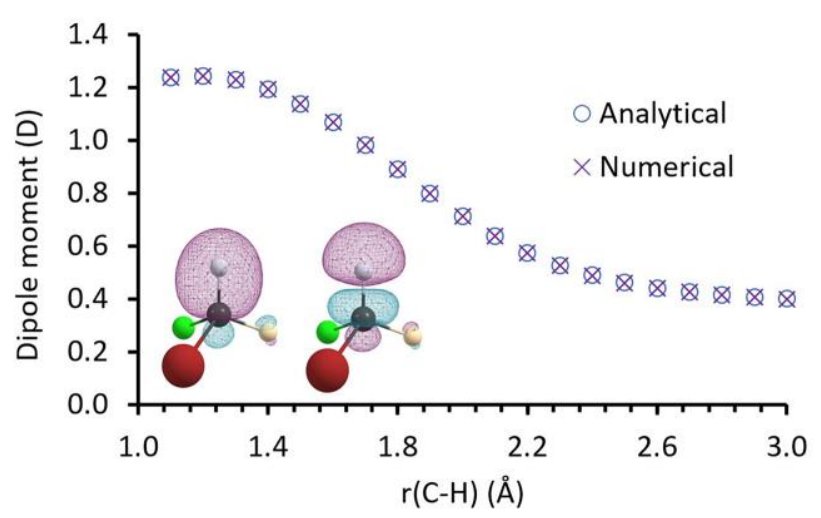

Figure 1. Analytical vs numerical MC-PDFT dipole moment curves of $\mathrm{CHFClBr}$. The curves are obtained by a rigid scan along the $\mathrm{C}-\mathrm{H}$ coordinate performed at the $\operatorname{tPBE}(2 \mathrm{e}, 2 \mathrm{o}) / \mathrm{aug}-\mathrm{cc}-\mathrm{pVDZ}$ level. The active space orbitals at the equilibrium geometry are shown in the inset.

\section{A. Equilibrium Dipole Moments of the Main-Group Polyatomic Species}

The electronic structure of closed-shell organic molecules is often well-described by a single electron configuration. However, the open-shell species, for example organic radicals and diradicals, are usually inherently multiconfigurational and may require a multireference treatment. We used tPBE to compute equilibrium dipole moments of the four highly reactive open-shell polyatomic species given in Table 1. In these species, the first atom was placed at the origin and the Al-C, C-P, C-C, and C-N bonds were aligned with the $z$-axis making the $x$ - and $y$-components of the dipole moment zero.

In the case of $\mathrm{AlCH}_{2}$, the CASSCF dipole moment $(0.84 \mathrm{D})$ is already close to the reference MRCISD value $(0.85 \mathrm{D})$, and tPBE introduces only a minor change (to $0.82 \mathrm{D})$, thereby avoiding an overcorrection. For the CPP, CCO, and CNN diradicals, the CASSCF dipole moments deviate significantly from the reference values. Here, tPBE largely improves upon CASSCF results by reducing the absolute error and even flipping the CASSCF dipole moment vector in the CPP diradical to yield the 
correct ${ }^{(-)} \mathrm{C}_{-} \mathrm{P}_{2}^{(+)}$polarity. Another inherently multiconfigurational molecule is $C$-cyanomethanimine. It can exist in the $E$ and $Z$ forms with quite different dipole moments of 4.11 and 1.41 D. ${ }^{57}$ Both CASSCF and $\mathrm{TPBE}$ can distinguish between the two isomers; however the CASSCF values are overestimated by almost $0.3 \mathrm{D}$, while tPBE dipole moments are in excellent agreement with the experimental references.

Table 1. CASSCF and tPBE dipole moments $(\mu)$ and projections on the $z$-axis $\left(\mu_{\mathrm{z}}\right)$ computed with the aug-cc-pVTZ basis set.

\begin{tabular}{|c|c|c|c|c|c|c|}
\hline Species & State & Active space & $M$ & CASSCF & tPBE & Reference \\
\hline \multicolumn{7}{|c|}{$\mu_{\mathrm{z}}(\mathrm{D})$} \\
\hline $\mathrm{AlCH}_{2}$ & ${ }^{2} \mathrm{~B}_{1}$ & $(9 \mathrm{e}, 10 \mathrm{o})$ & 0.10 & 0.84 & 0.82 & $0.85^{\mathrm{a}}$ \\
\hline CPP & ${ }^{3} \Pi$ & $(14 \mathrm{e}, 12 \mathrm{o})$ & 0.13 & -0.11 & 0.38 & $0.32^{\mathrm{b}}$ \\
\hline $\mathrm{CCO}$ & ${ }^{3} \Sigma^{-}$ & $(14 \mathrm{e}, 12 \mathrm{o})$ & 0.05 & 1.86 & 1.36 & $1.47^{\mathrm{b}}$ \\
\hline $\mathrm{CNN}$ & ${ }^{3} \Sigma^{-}$ & $(14 \mathrm{e}, 12 \mathrm{o})$ & 0.07 & 1.21 & 0.79 & $0.82^{\mathrm{b}}$ \\
\hline \multicolumn{7}{|c|}{$\mu(\mathrm{D})$} \\
\hline $\mathrm{HN}=\mathrm{CHCN}(E$ isomer $)$ & $A^{\prime}$ & (10e,10o) & 0.07 & 4.48 & 4.15 & $4.11(2)^{\mathrm{c}}$ \\
\hline $\mathrm{HN}=\mathrm{CHCN}(Z$ isomer $)$ & $A^{\prime}$ & $(10 \mathrm{e}, 10 \mathrm{o})$ & 0.08 & 1.71 & 1.43 & $1.41(17)^{\mathrm{c}}$ \\
\hline
\end{tabular}

\section{B. Dipole Moments at the Nonequilibrium Configurations}

Predicting the dipole moment for geometries with stretched bonds is a challenge for electronic structure theory. For example, coupled-cluster theory with single, double, and quasiperturbative connected triple excitations, $(\operatorname{CCSD}(\mathrm{T})),{ }^{61}$ which is often reliable at equilibrium geometries, can fail dramatically at large internuclear distances and give rise not only to inaccurate potential energy surfaces (PESs) but also to unphysical DMSs. ${ }^{62}$ This can be attributed to strong electron correlation at geometries with stretched bonds; treating strong correlation by using single-reference methods requires a more complete treatment of higher excitations, which is often unaffordable. ${ }^{63}$

To evaluate the performance of MC-PDFT for predicting dipole moment curves (DMCs), we chose four molecules (HF, NO, $\mathrm{CO}$, and $\mathrm{AlO}$ ) that have been extensively studied both experimentally and theoretically in the past. The diatomics were oriented along the $z$-axis with the first atom placed at the origin. Thus, the positive projection of the dipole moment on the $z$-axis implies ${ }^{-} \mathrm{AB}^{+}$polarity, while a negative value indicates the reverse ${ }^{-} \mathrm{AB}^{+}$polarity. The DMCs are scanned from distances slightly shorter than the equilibrium one all the way to the asymptotic limit when possible. The tPBE curves are compared to MRCISD+Q results with both methods built upon the same parent CASSCF wave functions. In addition to computed ab initio curves, we also show selected points of empirical DMCs available in the literature 
(where they are given in the form of polynomial series). These empirical DMCs were obtained from the relative intensities of rovibrational transitions using the Herman-Wallis approach ${ }^{64,65}$ or a direct-fit method by Li et al. ${ }^{66}$ where DMCs are fit to all available rovibrational matrix elements with appropriate weighting of experimental uncertainties. Because only low-energy vibrational bands $v^{\prime}-v^{\prime \prime}\left(v^{\prime}\right.$ is an upper and $v^{\prime \prime}$ is a lower state) can be unequivocally resolved in the emission spectra, the empirical DMCs represented by polynomial series are often limited to a short range of internuclear distances; the limits of the range correspond to the classical turning points of the highest vibrational level involved in the least-squares fitting. Nevertheless, empirical DMCs provide a valuable benchmark for the computed nonequilibrium dipole moments. Note that DMCs fitted to piecewise polynomial series diverge outside their validity range and are not used in practical calculations of rovibrational intensities; instead they are replaced with asymptotically correct analytical functions, for example a Padé approximant fitted to empirical data in the short range and to ab initio data at large internuclear separations in order to improve the intensities of high-overtone transitions. ${ }^{67}$ For our purposes, the accurate empirical DMCs in the form of polynomial series provide an opportunity to examine the quality of the nonequilibrium DMCs in the regions where predictions of single-reference electronic structure methods become questionable.

\section{Closed-Shell HF and CO Molecules}

In the $X^{1} \Sigma^{+}$ground state at the equilibrium bond length of $0.9168 \AA{ }^{68}$ the hydrogen fluoride wave function is dominated by a closed-shell $1 \sigma^{2} 2 \sigma^{2} 3 \sigma^{2} 1 \pi_{x}^{2} 1 \pi_{y}^{2}$ electron configuration. The polarity of HF is manifested by the permanent dipole moment of $1.8265 \mathrm{D}$, which can be rationalized from the difference in electronegativity of $\mathrm{H}$ (electronegativity 2.10) and $\mathrm{F}$ (electronegativity 3.98) atoms (Figure 2, left). ${ }^{69,70}$ This experimental value is reasonably well reproduced by all considered methods, including second-order corrected coupled cluster theory, $(\operatorname{CCSD}(2))^{71}$ extrapolated to the complete basis set limit by Hait and Head-Gordon. ${ }^{63}$ In that work, $\operatorname{CCSD}(2)$ was chosen as a benchmark to show the failure of density functional theory (DFT) at moderate and large internuclear separations, where the wave function acquires a sizable multiconfigurational character mainly due to the increasing contribution of the $1 \sigma^{2} 2 \sigma^{2} 4 \sigma^{2} 1 \pi_{x}^{2} 1 \pi_{y}^{2}$ electron configuration. Indeed, the $\operatorname{CCSD}(2)$ curve is smooth and shows the correct asymptotic behavior in the homolytic bond dissociation limit. However, a comparison with the empirical DMCs and full-valence (8e,5o) MRCISD+Q curves, which are in good agreement with each other, suggests that $\operatorname{CCSD(2)}$ is not a reliable benchmark as it starts to overestimate the dipole moment magnitude beyond $1.2 \AA$. Importantly, at larger internuclear distances, the $\operatorname{CCSD}(2)$ curve approaches 
zero much faster than the one predicted by MRCISD+Q with the two curves crossing one another around $2.1 \AA$. The discrepancy between $\operatorname{CCSD}(2)$ and MRCISD+Q cannot be explained by incompleteness of the basis set because an increase from aug-cc-pVQZ to aug-cc-pV5Z has only a small effect on the MRCISD+Q DMC (less than 0.01 D at all geometries). The most accurate empirical DMC of CO in the range from 0.69 to $1.39 \AA$ has been obtained by Li et $a l .^{22}$ based on the relative intensities of rovibrational bands with $v^{\prime} \leq 5$ and using the potential energy curve ${ }^{72}$ derived by Coxon and Hajigeorgiou. An alternative but older empirical DMC by Sileo and $\mathrm{Cool}^{73}$ extends to $1.7 \AA$ due to the use of transitions from upper vibrational levels $v^{\prime} \leq 9$; however, it has a greater uncertainty $(\leq 15 \%)$ in the fitted rovibrational matrix elements. Nevertheless, this curve overlaps quite well with MRCISD+Q and DMC by Li et al. This overlap provides some support for using the MRCISD+Q results as a benchmark.

According to full-valence MRCISD+Q, the HF polarity increases upon bond extension with the maximum at about $1.60 \AA$, and then it slowly reduces to zero in the bond dissociation limit (Figure 2, left). At an internuclear distance of $1.0 \AA, \operatorname{CASSCF}(8 \mathrm{e}, 5 \mathrm{o})$ and $\operatorname{tPBE}(8 \mathrm{e}, 5 \mathrm{o})$ results begin to deviate from the MRCISD+Q curve. The tPBE(8e,5o) DMC improves noticeably upon the CASSCF(8e,5o) results and yields a DMC that is much closer to the benchmark. The full-valence (8e,5o) active space has only one correlating orbital, and the populations of $2 \sigma, 1 \pi_{x}$, and $1 \pi_{y}$ orbitals do not change as functions of internuclear distance, so the DMC is virtually identical to the one obtained with the small (2e,2o) active space. The expansion of active space to $(8 \mathrm{e}, 8 \mathrm{o})$ by adding $2 \pi_{x}, 2 \pi_{y}$, and $5 \sigma$ orbitals with $3 \mathrm{p}_{\mathrm{x}}, 3 \mathrm{p}_{\mathrm{y}}$, and $3 \mathrm{~s}$ character reduces the magnitude of the MRCISD+Q DMC dipole moment but only slightly (by no more than $0.01 \mathrm{D})$. The maximum magnitude in the $\operatorname{tPBE}(8 \mathrm{e}, 8 \mathrm{o}) \mathrm{DMC}$ is shifted from 1.6 to $1.8 \AA$, which results in better agreement with the MRCISD+Q curve out to $1.5 \AA$ (see the SI). The CASSCF DMC for the larger active space also becomes closer to the benchmark. For the full-valence active space, the magnitude of the dipole moment across all geometries is underestimated by CASSCF and overestimated by tPBE with the largest deviation in the vicinity of the Coulson-Fischer point. However, this discrepancy with MRCISD+Q is largely eliminated by the $\mathrm{PBE} 0$ curve that is closer to the benchmark at the inflection point and almost overlapping with it in the other regions. This result is encouraging since the computational cost of tPBE0 is the same as $\mathrm{PBE}$ and much less than that of MRCISD+Q. 

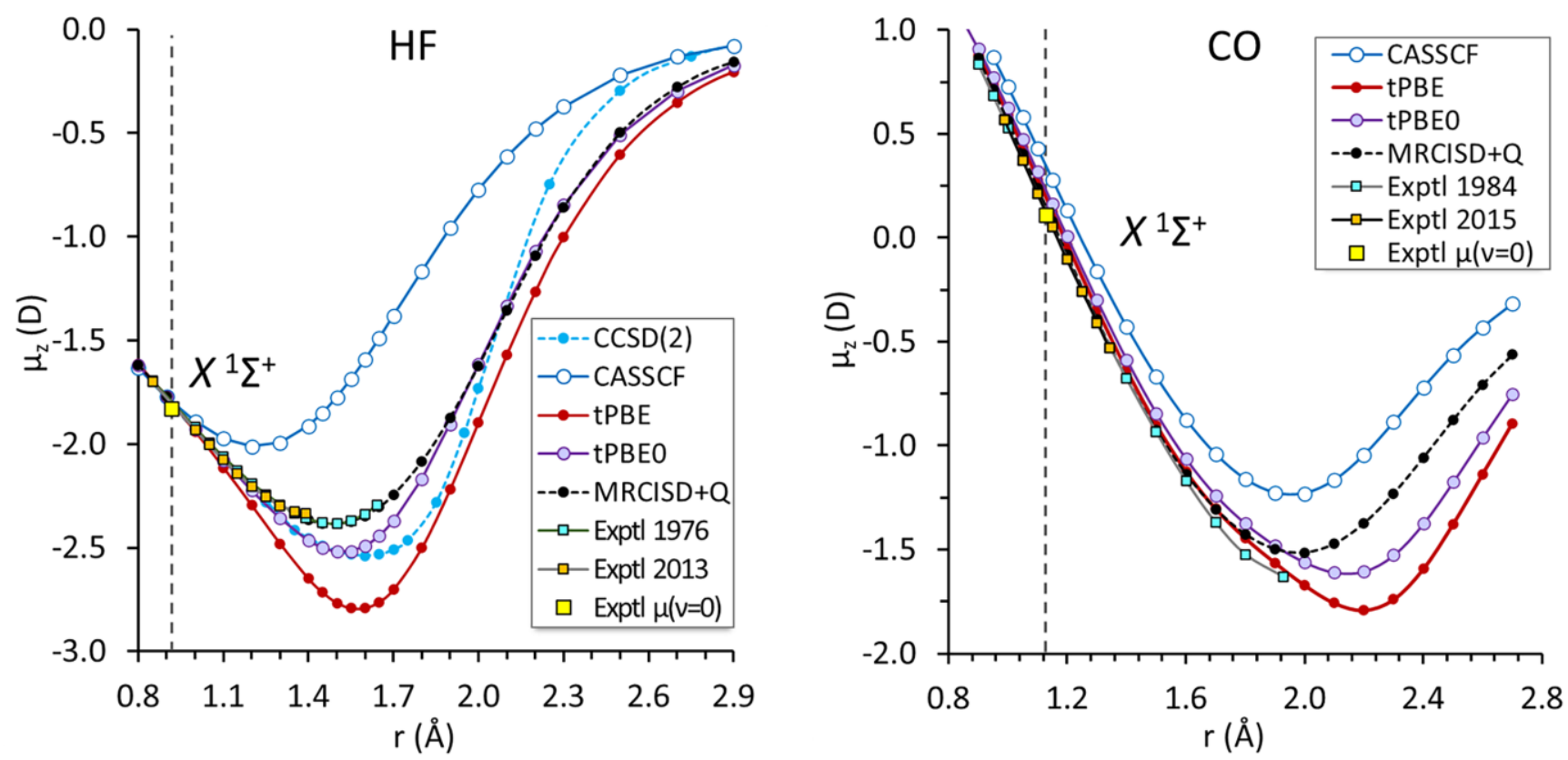

Figure 2. Dipole moment curves of HF (left) and CO (right) molecules. The vertical dashed lines mark the equilibrium internuclear distances. The active spaces are full-valence active spaces, in particular $(8 \mathrm{e}, 5 \mathrm{o})$ for $\mathrm{HF}$ and $(10 \mathrm{e}, 8 \mathrm{o})$ for $\mathrm{CO}$. Negative values of the dipole moment correspond to ${ }^{+} \mathrm{AB}^{-}$polarity. Yellow squares indicate dipole moments measured for the ground vibrational state. The orange $\left(\mathrm{HF},{ }^{22}\right.$ $\left.\mathrm{CO}^{74}\right)$ and cyan $\left(\mathrm{HF}^{73}, \mathrm{CO}^{75}\right)$ squares are the selected points of the empirical dipole moment curves within the validity range. The aug-cc-pVQZ basis set is used for all calculations except for the CCSD(2)/CBS calculations, which are taken from ref ${ }^{63}$.

The dominant configuration of the ${ }^{1} \Sigma^{+}$ground state of carbon monoxide is $3 \sigma^{2} 4 \sigma^{2} 5 \sigma^{2} 1 \pi_{x}^{2} 1 \pi_{y}^{2}$. In the vicinity of equilibrium geometry ${ }^{76}$ at $1.1283 \AA$, all methods reproduce the counterintuitive ${ }^{-} \mathrm{CO}^{+}$ polarity with the magnitude of dipole moment close to the experimental ${ }^{77}$ value of $0.112(5) \mathrm{D}$. Such distribution of electron density arises from the donation of the $2 p(\mathrm{O})$ electron pair back to the $\mathrm{C}$ atom. As the internuclear distance increases, the polarity changes to ${ }^{+} \mathrm{CO}^{-}$reflecting the migration of the electronic charge. The empirical DMC of the CO molecule has been a subject of numerous experimental studies in the past. ${ }^{75,78-82}$ To date, the most accurate empirical DMC is a six-order polynomial constructed by Li et $a l{ }^{74}$ based on the intensity ratios of lines ascribed to transitions from the upper states with $v^{\prime} \leq 6$ and spectroscopically determined potential energy curve ${ }^{76}$ obtained by Coxon and Hajigeorgiou. This DMC spans a range of nuclear oscillations from 0.99 to $1.34 \AA$ corresponding to the turning points of the $6^{\text {th }}$ vibrational level. The older and less accurate DMC by Chackerian et al., relying on the data available before 1984 , covers a substantially larger range from 0.87 to $1.93 \AA$ and almost reaches the inflection point of full-valence (10e,8o) MRCISD+Q curve around 2.0 $\AA .{ }^{75}$ In general, these empirical DMCs are in good agreement with MRCISD+Q and the previous study by Langhoff and Bauschlicher, ${ }^{83}$ who used 
$\operatorname{ACPF}(8 \mathrm{e}, 7 \mathrm{o}) / \mathrm{aug}-\mathrm{cc}-\mathrm{pVQZ}$ level of theory for calculating the DMC of CO molecule. The noticeable deviation of DMC by Chackerian et al. from MRCISD+Q between 1.6 and $1.9 \AA$ can be attributed to the increasing uncertainty of experimental values. In contrast to HF, the state-specific DMC of CO is a smooth function of distance only up to $2.7 \AA$. Beyond this point, the $X^{1} \Sigma^{+}$and $B^{1} \Sigma^{+}$states become near-degenerate leading to the root-flipping problem (see SI). Note that, the first excited singlet state $A^{1} \Pi$ also belongs to the same dissociation channel, ${ }^{84}$ however it does not mix with the ${ }^{1} \Sigma^{+}$states because of the different symmetry. The tPBE DMC agrees very well with the benchmark values up to $1.8 \AA$ and overestimates the dipole magnitude at longer internuclear distances (Figure 2, right). The maximum absolute value is shifted toward 2.3 Å compared to 2.0 Å for MRCISD+Q and CASSCF DMCs. The tPBE0 improves upon tPBE at the distances greater than $2.0 \AA$ but slightly degrades at shorter distances.

\section{Open-Shell NO and AlO Radicals}

In contrast to $\mathrm{HF}$ and $\mathrm{CO}$, nitric oxide is a radical; it has a degenerate ground electronic state of ${ }^{2} \Pi$ symmetry with an unpaired electron occupying the $1 \pi_{x}$ or $1 \pi_{y}$ orbital. The experimental dipole moment ${ }^{85}$ is small, $0.1595(15) \mathrm{D}$, with $\mathrm{O}$ positive. The formal bond order is 2.5 , making the bond slightly shorter than in $\mathrm{CO}$; the equilibrium bond distance is $1.1508 \AA .{ }^{86}$ In the ground state, the sign of the dipole moment of NO changes upon bond extension as the molecule dissociates into $\mathrm{N}\left({ }^{4} \mathrm{~S}\right)$ and $\mathrm{O}\left({ }^{3} \mathrm{P}\right)$ atoms. The empirical DMC obtained by Bood et al. ${ }^{86}$ covers the range from 0.91 to $1.74 \AA$ and overlaps well with the full-valence (11e,8o) MRCISD+Q benchmark. As for HF and CO (Figure 2), the tPBE dipole moment curve for NO (Figure 3) overestimates the magnitude of the MRCISD+Q dipole moment in the vicinity of the inflection point, while it provides accurate values at the equilibrium and moderate elongations and leads to a correct asymptotic decay. A significant improvement is achieved by tPBE0, which reduces deviation not only at the DMC minimum but also at the large internuclear distances. All three methods, MRCISD+Q, tPBE and tPBE0, predict the maximum polarity to be at $1.85 \AA$, while the CASSCF value is shifted toward a shorter internuclear distance of $1.7 \AA$. 

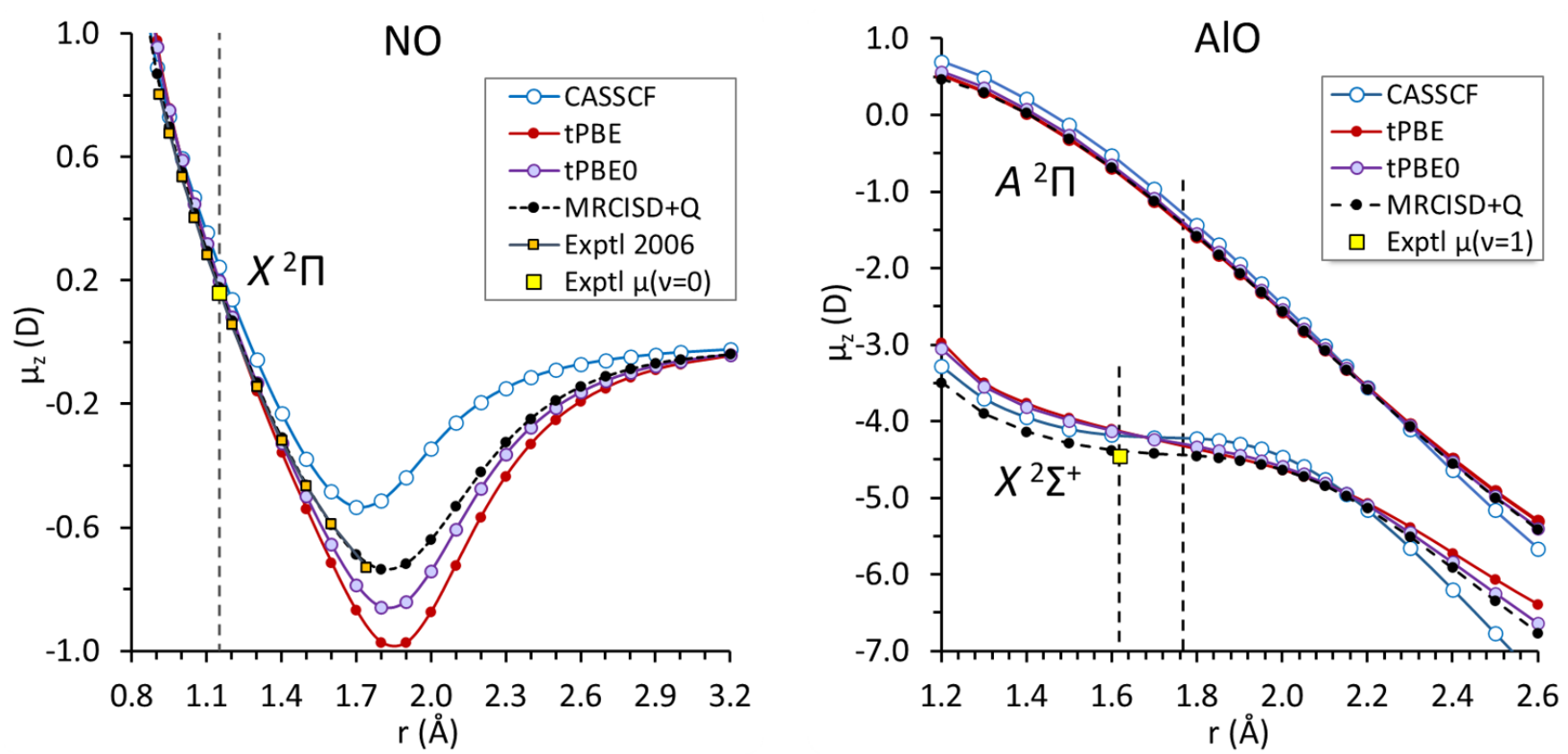

Figure 3. Dipole moment curves of NO (left) and AlO (right). The vertical dashed lines mark the equilibrium internuclear distances. The active space is the full-valence one for NO, which is (11e,8o). The active space for $\mathrm{AlO}$ is $(7 \mathrm{e}, 10 \mathrm{o})$. Positive values correspond to ${ }^{-} \mathrm{AB}^{+}$polarity. Yellow squares indicate dipole moments measured for the indicated vibrational state $v$. The orange squares are selected points of the NO empirical dipole moment curve ${ }^{87}$ within its validity range. The aug-cc-pVQZ basis set is used for all calculations in this figure.

The AlO radical has a $X^{2} \Sigma^{+}$nondegenerate ground electronic state with a singly occupied $7 \sigma$ orbital. In the lowest ${ }^{2} \Pi$ excited state, the bond is elongated to $1.7678 \AA .{ }^{88}$ While there is no empirical DMC for this radical, the permanent dipole moment of 4.45(3) D has recently been measured for the ${ }^{2} \Sigma^{+}$ $(v=1)$ state using microwave Stark spectroscopy. ${ }^{89}$ The computed DMCs of the lowest two electronic states of $\mathrm{AlO},{ }^{2} \Sigma^{+}$and ${ }^{2} \Pi$, are shown in Figure 3.

At the equilibrium bond length ${ }^{90}$ of $1.6178 \AA$, the wave function of the ground state is dominated by the $5 \sigma^{2} 6 \sigma^{2} 2 \pi_{x}^{2} 2 \pi_{y}^{2} 7 \sigma^{1}$ configuration with a significant contribution of the $5 \sigma^{2} 6 \sigma^{1} 2 \pi_{x}^{2} 2 \pi_{y}^{2} 7 \sigma^{2}$ configuration. To try to better describe the contributions of the $\mathrm{Al}^{+} \mathrm{O}^{-}$and $\mathrm{Al}^{2+} \mathrm{O}^{2-}$ oxidation states, we enlarged the $(9 \mathrm{e}, 8 \mathrm{o})$ full-valence valence active space $(9 \mathrm{e}, 8 \mathrm{o})$ by including an additional set of $p$ orbitals of the $\mathrm{O}$ atom $\left(4 \pi_{x}, 4 \pi_{y}\right.$, and $\left.9 \sigma\right)$ into as was suggested in some previous works. ${ }^{91,92}$ However, we kept the low-lying $5 \sigma$ orbital doubly occupied in all configurations to prevent its orbital rotation and increase the SCF stability; this yields a (7e10o) active space. The corresponding tPBE and tPBE0 curves practically overlap with the MRCISD+Q benchmark for the ${ }^{2} \Pi$ state and the three curves are also in reasonable agreement for the ${ }^{2} \Sigma^{+}$state. 


\section{First-Row Transition Metal Diatomics}

Transition metal diatomics display remarkably rich and complex electronic spectra due to the partially occupied $d$ subshell of the metal. The dense manifolds of electronic states in such molecules often lead to problematic energy convergence and difficulty in obtaining a solution of the proper symmetry. An example is nickel hydride, $\mathrm{NiH}$, where the adiabatic gap between the ground ${ }^{1} \Delta$ and excited ${ }^{1} \Sigma^{+}$states calculated by $(9 \mathrm{e}, 10 \mathrm{o}) \mathrm{MRCISD+Q} / \mathrm{def} 2-\mathrm{TZVPD}$ is only $6.9 \mathrm{kcal} / \mathrm{mol}$. In $\mathrm{C}_{2 \mathrm{v}}$ symmetry, geometry optimization of $\mathrm{NiH}$ starting from a poor $\mathrm{A}_{1}$ initial guess may result in the formation of the excited state ${ }^{1} \Sigma^{+}\left({ }^{1} \mathrm{~A}_{1}\right)$ rather than the ground state ${ }^{1} \Delta\left({ }^{1} \mathrm{~A}_{1}+{ }^{1} \mathrm{~A}_{2}\right)$ since both states have $\mathrm{A}_{1}$ components. Despite the small energy separation, the predicted equilibrium dipole moments of these two states, 2.81 $\mathrm{D}\left({ }^{1} \Delta\right)$ and $3.56 \mathrm{D}\left({ }^{1} \Sigma^{+}\right)$, are noticeably different. An even larger difference was found between the dipole moments of the well-separated $X^{1} \Delta$ and $B{ }^{1} \Delta$ state, for which the experimental values are 2.44(2) and $0.36(2) \mathrm{D}$, respectively. Thus, it is crucial to obtain the correct symmetry of the wave function together with the correct state ordering when computing the dipole moments.

To evaluate the performance of MC-PDFT for predicting dipole moments, we selected 18 diatomic molecules comprised of a first-row transition metal and a main-group element for which experimental values are available in the literature (Table 2). The ground state dipole moments were mostly taken from the datasets of Steimle ${ }^{93}$ and Liu et al., ${ }^{94}$ who give the original experimental references. We also added $\mathrm{ScF},{ }^{95} \mathrm{ScH},{ }^{96}$ and $\mathrm{VS}^{97}$ molecules to our dataset. The old reference ${ }^{98}$ for $\mathrm{TiO}$ was replaced with the more recent experimental value of 3.34(1) D. ${ }^{99}$ The dipole moment of $\mathrm{CrH}$ was estimated to be 3.50(3) D based on the dependence of rovibrational spectrum of $\mathrm{CrD}$ on the applied electric field. ${ }^{100}$ Note that this value is also in excellent agreement with $3.51 \mathrm{D}$ computed at the $(7 \mathrm{e}, 18 \mathrm{o}) \mathrm{MRCISD+Q} / \mathrm{aug}-\mathrm{cc}-\mathrm{pVQZ}-\mathrm{DK}$ level. ${ }^{101}$ In the case of $\mathrm{ScO}$, we used a reference ${ }^{102}$ of $3.67 \mathrm{D}$ obtained with (7e12o)MRCISD+Q/aug-ccpVQZ+DKH2 rather than the experimental value ${ }^{103}$ of 4.55(8) D that also largely contradicts $3.81 \mathrm{D}$ obtained with CCSD(T)/aug-cc-pVQZ+DKH. ${ }^{102}$ Similarly, in the case of $\mathrm{CoH}\left({ }^{3} \Phi\right)$, instead of using the experimental value ${ }^{104}$ of $1.88(8) \mathrm{D}$, which is likely to be overestimated, we used the best theoretical estimate of $2.63 \mathrm{D}$, which was independently predicted by two multireference methods, (18e,14o)MRCISD+Q/5 $\zeta^{105}$ and internally contracted (10e,7o)MRCCSD/cc-pVTZ-DK. ${ }^{106}$ The equilibrium bond lengths were taken from Aoto et al. ${ }^{107}$ and references therein, except for $\mathrm{FeC}^{108}$ and $\mathrm{CoH}^{109,110}$ with revised bond lengths of $1.589 \AA$ and $1.514 \AA$, respectively. Also, we used the best estimate of the bond length in FeH of $1.561 \AA$ obtained by CCSDT in the complete basis set limit of the aug-cc- 
pwCVnZ-DK series. ${ }^{111}$ Finally, we neglected vibrational averaging ${ }^{112,113}$ when comparing the computed and measured dipole moments because this correction is usually small with a typical error of a few percent, ${ }^{63}$ which is often comparable to the uncertainty of experimental values and incompleteness of the basis set.

Most of the molecules in our dataset are open-shell species with only two singlet molecules, $\mathrm{ScF}$ and $\mathrm{ScH}$. The sizes of the active spaces and orbital types are summarized in Table 2. In addition to that, we also report the weights of the leading configurations and multireference $M$ diagnostic parameters computed at the mod-CPO level with fixed experimental bond lengths. Molecules with $M$ diagnostics greater than 0.05 or weights lower than $95 \%$ are considered to be strongly correlated (inherently multiconfigurational molecules for which a multireference treatment would be recommended); it turns out that 17 of the molecules fall in this category, with the only exception being ScF. In Table $2, \sigma$ and $\pi$ denote bonding orbitals of $3 d \sigma$ and $3 d \pi$ character with nonmetal $n \mathrm{p} \sigma$ and $n \mathrm{p} \pi$ contributions, respectively. The $3 d$ label refers to the $3 d \pi$ or $3 d \delta$ orbitals, $4 s$ implies the back-polarized $4 s / 4 p$ hybrid orbitals, and $2 s$ and $3 s$ denote a lone-pair orbital of the non-metal. The extension of the nom-CPO to mod-CPO increases the active space in 7 out of 18 considered molecules. The ext-CPO active space is significantly larger than the nom-CPO and mod-CPO and embraces all valence electrons for the studied molecules. We used orbitals of the $4 d$-subshell to correlate $3 d$ occupied orbitals and $3 s$ and $4 s$ lone-pair orbitals to correlate the $2 s$ and $3 s$ orbitals of the non-metal, respectively. The character of the correlating $4 s^{\prime}$ does not necessarily correspond to an $s$-type orbitals (see the natural orbitals in the SI).

Table 2. Definition of nominal and moderate CPO active spaces. The nom-CPO column provides a full list of orbitals in nom-CPO active space, whereas the mod-CPO column shows orbitals added to nom$\mathrm{CPO}$ to form the mod-CPO active space. The prime symbols indicate correlating orbitals. The orbitals in bold are formally singly occupied.

\begin{tabular}{|c|c|c|c|c|c|c|c|c|}
\hline \multirow{2}{*}{ Species } & \multirow{2}{*}{ State } & \multirow{2}{*}{ Weight } & \multirow{2}{*}{$M$} & \multicolumn{2}{|c|}{ Active space size } & \multicolumn{2}{|l|}{ Orbital type } & \multirow{2}{*}{$\begin{array}{l}\text { Dominant electron } \\
\text { configuration }\end{array}$} \\
\hline & & & & $\begin{array}{l}\text { nom- } \\
\mathrm{CPO}\end{array}$ & $\begin{array}{l}\text { mod- } \\
\mathrm{CPO}\end{array}$ & nom-CPO & mod-CPO & \\
\hline $\mathrm{CoH}$ & ${ }^{3} \Phi$ & $48 \%$ & 0.99 & $(4 \mathrm{e}, 60)$ & $(8 \mathrm{e}, 10 \mathrm{o})$ & $\left(\sigma, \sigma^{*}\right), \mathbf{2}\left(\mathbf{3 d}, \mathbf{3} \mathbf{d}^{\prime}\right)$ & $2\left(3 d, 3 d^{\prime}\right)$ & $6 \sigma^{2} 7 \sigma^{2} 3 \pi^{3} 1 \delta^{3}$ \\
\hline $\mathrm{CrH}$ & ${ }^{6} \Sigma^{+}$ & $94 \%$ & 0.09 & $(7 e, 120)$ & $(7 \mathrm{e}, 12 \mathrm{o})$ & $\left(\sigma, \sigma^{*}\right), \mathbf{4}\left(\mathbf{3 d}, \mathbf{3} d^{\prime}\right),\left(\mathbf{4 s}, \mathbf{4} \mathbf{s}^{\prime}\right)$ & None & $6 \sigma^{2} 7 \sigma^{1} 3 \pi_{x}^{1} 3 \pi_{y}^{1} 1 \delta^{2}$ \\
\hline $\mathrm{CrN}$ & ${ }^{4} \Sigma^{-}$ & $76 \%$ & 0.16 & $(9 \mathrm{e}, 12 \mathrm{o})$ & $(9 \mathrm{e}, 12 \mathrm{o})$ & $\left(\sigma, \sigma^{*}\right), 2\left(\pi, \pi^{*}\right),\left(\mathbf{4 s}, \mathbf{4} \mathbf{s}^{\prime}\right), \mathbf{2}\left(\mathbf{3 d}, \mathbf{3} \mathbf{d}^{\prime}\right)$ & None & $8 \sigma^{2} 9 \sigma^{1} 3 \pi_{x}^{2} 3 \pi_{y}^{2} 1 \delta^{2}$ \\
\hline $\mathrm{CrO}$ & ${ }^{5} \Pi$ & $84 \%$ & 0.1 & $(10 \mathrm{e}, 14 \mathrm{o})$ & $(10 \mathrm{e}, 14 \mathrm{o})$ & $\left(\sigma, \sigma^{*}\right), 2\left(\pi, \pi^{*}\right),\left(\mathbf{4} \mathbf{s}, \mathbf{4} \mathbf{s}^{\prime}\right), \mathbf{3}\left(\mathbf{3 d}, \mathbf{3} \mathbf{d}^{\prime}\right)$ & None & $8 \sigma^{2} 9 \sigma^{2} 10 \sigma^{1} 2 \pi^{4} 3 \pi^{1} 1 \delta^{2}$ \\
\hline $\mathrm{FeC}$ & ${ }^{3} \Delta$ & $72 \%$ & 0.20 & $(8 \mathrm{e}, 10 \mathrm{o})$ & $(10 \mathrm{e}, 12 \mathrm{o})$ & $\left(\sigma, \sigma^{*}\right), 2\left(\pi, \pi^{*}\right),\left(\mathbf{4} \mathbf{s}, \mathbf{4} \mathbf{s}^{\prime}\right),\left(\mathbf{3 d}, \mathbf{3} \mathbf{d}^{\prime}\right)$ & $\left(3 \mathrm{~d}, 3 \mathrm{~d}^{\prime}\right)$ & $7 \sigma^{2} 8 \sigma^{2} 3 \pi_{x}^{2} 3 \pi_{y}^{2} 9 \sigma^{1} 1 \delta^{3}$ \\
\hline $\mathrm{FeH}$ & ${ }^{4} \Delta$ & $94 \%$ & 0.05 & $(5 \mathrm{e}, 8 \mathrm{o})$ & $(7 \mathrm{e}, 10 \mathrm{o})$ & $\left(\sigma, \sigma^{*}\right), \mathbf{3}\left(\mathbf{3 d} \mathbf{d}, \mathbf{3} \mathbf{d}^{\prime}\right)$ & $\left(3 \mathrm{~d}, 3 \mathrm{~d}^{\prime}\right)$ & $6 \sigma^{2} 7 \sigma^{2} 3 \pi_{x}^{1} 3 \pi_{y}^{1} 1 \delta^{3}$ \\
\hline $\mathrm{NiH}$ & ${ }^{2} \Delta$ & $93 \%$ & 0.05 & $(3 \mathrm{e}, 4 \mathrm{o})$ & $(9 \mathrm{e}, 10 \mathrm{o})$ & $\left(\sigma, \sigma^{*}\right),\left(\mathbf{3 d}, \mathbf{3} \mathbf{d}^{\prime}\right)$ & $3\left(3 \mathrm{~d}, 3 \mathrm{~d}^{\prime}\right)$ & $6 \sigma^{2} 7 \sigma^{2} 3 \pi_{x}^{2} 3 \pi_{y}^{2} 1 \delta^{3}$ \\
\hline
\end{tabular}




\begin{tabular}{|c|c|c|c|c|c|c|c|}
\hline $\mathrm{ScF}$ & ${ }^{1} \Sigma^{+}$ & $97 \%$ & $0.02(2 \mathrm{e}, 2 \mathrm{o})$ & $(6 e, 60)$ & $\left(\sigma, \sigma^{*}\right)$ & $2\left(\mathrm{p}, \mathrm{p}^{\prime}\right)$ & $7 \sigma^{2} 8 \sigma^{2} 9 \sigma^{2} 3 \pi_{x}^{2} 3 \pi_{y}^{2}$ \\
\hline $\mathrm{ScH}$ & ${ }^{1} \Sigma^{+}$ & $90 \%$ & $0.17(2 \mathrm{e}, 2 \mathrm{o})$ & $(4 \mathrm{e}, 4 \mathrm{o})$ & $\left(\sigma, \sigma^{*}\right)$ & $\left(3 \mathrm{~d}, 3 \mathrm{~d}^{\prime}\right)$ & $6 \sigma^{2} 7 \sigma^{2}$ \\
\hline $\mathrm{ScO}$ & ${ }^{2} \Sigma^{+}$ & $93 \%$ & $0.05(7 \mathrm{e}, 8 \mathrm{o})$ & $(7 \mathrm{e}, 8 \mathrm{o})$ & $\left(\sigma, \sigma^{*}\right), 2\left(\pi, \pi^{*}\right),\left(\mathbf{4 s}, \mathbf{4} \mathbf{s}^{\prime}\right)$ & None & $7 \sigma^{2} 8 \sigma^{2} 9 \sigma^{1} 3 \pi_{x}^{2} 3 \pi_{y}^{2}$ \\
\hline $\mathrm{ScS}$ & ${ }^{2} \Sigma^{+}$ & $92 \%$ & $0.05(7 \mathrm{e}, 8 \mathrm{o})$ & $(7 e, 80)$ & $\left(\sigma, \sigma^{*}\right), 2\left(\pi, \pi^{*}\right),\left(\mathbf{4 s}, \mathbf{4 \mathbf { s } ^ { \prime }}\right)$ & None & $9 \sigma^{2} 10 \sigma^{2} 11 \sigma^{1} 4 \pi_{x}^{2} 4 \pi_{y}^{2}$ \\
\hline $\mathrm{TiH}$ & ${ }^{4} \Phi$ & $49 \%$ & $0.78(5 \mathrm{e}, 8 \mathrm{o})$ & $(5 \mathrm{e}, 12 \mathrm{o})$ & $\left(\sigma, \sigma^{*}\right),\left(\mathbf{4 s}, \mathbf{4} \mathbf{s}^{\prime}\right), \mathbf{2}\left(\mathbf{3 d}, \mathbf{3} \mathbf{d}^{\prime}\right)$ & $2\left(3 \mathrm{~d}, 3 \mathrm{~d}^{\prime}\right)$ & $6 \sigma^{2} 8 \sigma^{1} 3 \pi^{1} 1 \delta^{1}$ \\
\hline TiN & ${ }^{2} \Sigma^{+}$ & $88 \%$ & $0.09(7 \mathrm{e}, 8 \mathrm{o})$ & $(7 \mathrm{e}, 8 \mathrm{o})$ & $\left(\sigma, \sigma^{*}\right), 2\left(\pi, \pi^{*}\right),\left(\mathbf{4 s}, \mathbf{4} \mathbf{s}^{\prime}\right)$ & None & $7 \sigma^{2} 8 \sigma^{2} 9 \sigma^{1} 3 \pi_{x}^{2} 3 \pi_{y}^{2}$ \\
\hline $\mathrm{TiO}$ & ${ }^{3} \Delta$ & $92 \%$ & $0.07(8 \mathrm{e}, 10 \mathrm{o})$ & $(8 \mathrm{e}, 10 \mathrm{o})$ & $\left(\sigma, \sigma^{*}\right), 2\left(\pi, \pi^{*}\right),\left(\mathbf{4 s}, \mathbf{4} \mathbf{s}^{\prime}\right),\left(\mathbf{3 d}, \mathbf{3} \mathbf{d}^{\prime}\right)$ & None & $7 \sigma^{2} 8 \sigma^{2} 3 \pi^{4} 1 \delta^{1} 9 \sigma^{1}$ \\
\hline TiS & ${ }^{3} \Delta$ & $89 \%$ & $0.09(8 \mathrm{e}, 10 \mathrm{o})$ & $(8 \mathrm{e}, 10 \mathrm{o})$ & $\left(\sigma, \sigma^{*}\right), 2\left(\pi, \pi^{*}\right),\left(\mathbf{3 d}, \mathbf{3} \mathbf{d}^{\prime}\right),\left(\mathbf{4 s}, \mathbf{4} \mathbf{s}^{\prime}\right)$ & None & $9 \sigma^{2} 10 \sigma^{2} 4 \pi^{4} 1 \delta^{1} 11 \sigma^{1}$ \\
\hline $\mathrm{VN}$ & ${ }^{3} \Delta$ & $84 \%$ & $0.11(8 \mathrm{e}, 10 \mathrm{o})$ & $(8 \mathrm{e}, 10 \mathrm{o})$ & $\left(\sigma, \sigma^{*}\right), 2\left(\pi, \pi^{*}\right), \mathbf{2}\left(\mathbf{3 d}, \mathbf{3} \mathbf{d}^{\prime}\right)$ & None & $7 \sigma^{2} 8 \sigma^{2} 9 \sigma^{1} 3 \pi_{x}^{2} 3 \pi_{y}^{2} 1 \delta^{1}$ \\
\hline vo & ${ }^{4} \Sigma^{-}$ & $89 \%$ & $0.08(9 \mathrm{e}, 12 \mathrm{o})$ & $(9 \mathrm{e}, 12 \mathrm{o})$ & $\left(\sigma, \sigma^{*}\right), 2\left(\pi, \pi^{*}\right), \mathbf{2}\left(\mathbf{3 d}, \mathbf{3} \mathbf{d}^{\prime}\right),\left(\mathbf{4 s}, \mathbf{4} \mathbf{s}^{\prime}\right)$ & None & $7 \sigma^{2} 8 \sigma^{2} 9 \sigma^{1} 3 \pi_{x}^{2} 3 \pi_{y}^{2} 1 \delta^{2}$ \\
\hline VS & ${ }^{4} \Sigma^{-}$ & $84 \%$ & $0.11(9 \mathrm{e}, 12 \mathrm{o})$ & $(9 \mathrm{e}, 12 \mathrm{o})$ & $\left(\sigma, \sigma^{*}\right), 2\left(\pi, \pi^{*}\right), 2\left(\mathbf{3 d}, 3 d^{\prime}\right),\left(4 \mathbf{s}, 4 \mathbf{s}^{\prime}\right)$ & None & $9 \sigma^{2} 10 \sigma^{2} 11 \sigma^{1} 4 \pi_{x}^{2} 4 \pi_{y}^{2} 1 \delta^{2}$ \\
\hline
\end{tabular}

Unlike the previous definition of the mod-CPO for transition metals,${ }^{51}$ here we extend it by adding to the nom-CPO active space not only the doubly occupied $3 d$ and $3 d^{\prime}$ orbitals but also the formally empty $d$-shell orbitals and their correlated counterparts for species with the high spatial symmetries, $\Phi$ and $\Gamma$. Note that these extra orbitals are unoccupied only in the single-reference framework, while they have substantial occupations in the multireference framework. This enhancement allows capturing the prominent multiconfigurational character of $\mathrm{TiH}\left({ }^{4} \Phi\right)$ that would be impossible to handle in the original formulation of mod-CPO. In the nom-CPO (5e8o) active space, the $M$ multireference diagnostic is only 0.09 and the leading determinant has a weight of $92 \%$. However, the expansion of the active space to mod-CPO (5e,12o) dramatically increases $M$ to 0.78 and results in a significant reduction of the leading determinant weight to $49 \%$, which are clear indications of the multiconfigurational character of this molecule. In the case of $\mathrm{CoH}$, all doubly occupied $d$-shell orbitals are also required to obtain the correct ground state ${ }^{3} \Phi$ with the $M$ value of 0.99 at the mod-CPO level. The wave function is dominated by the doubly-degenerate open-shell configurations $6 \sigma^{2} 7 \sigma^{2} 2 \pi^{4} 3 \pi^{3} 1 \delta^{3}$ each having a weight of $47 \%$. The multiconfigurational character of the $\mathrm{CoH}$ wave function is also evident from the partial occupation numbers of $3 \pi$ and $1 \delta$ natural orbitals illustrated in the left panel of Figure 4, for mod-CPO $(8 \mathrm{e}, 10 \mathrm{o})$ and ext-CPO $(10 \mathrm{e}, 12 \mathrm{o})$ active spaces. The nom-CPO active space is too small to capture $\sim 1.5$ occupations and therefore the mod-CPO and ext-CPO should be preferred. 


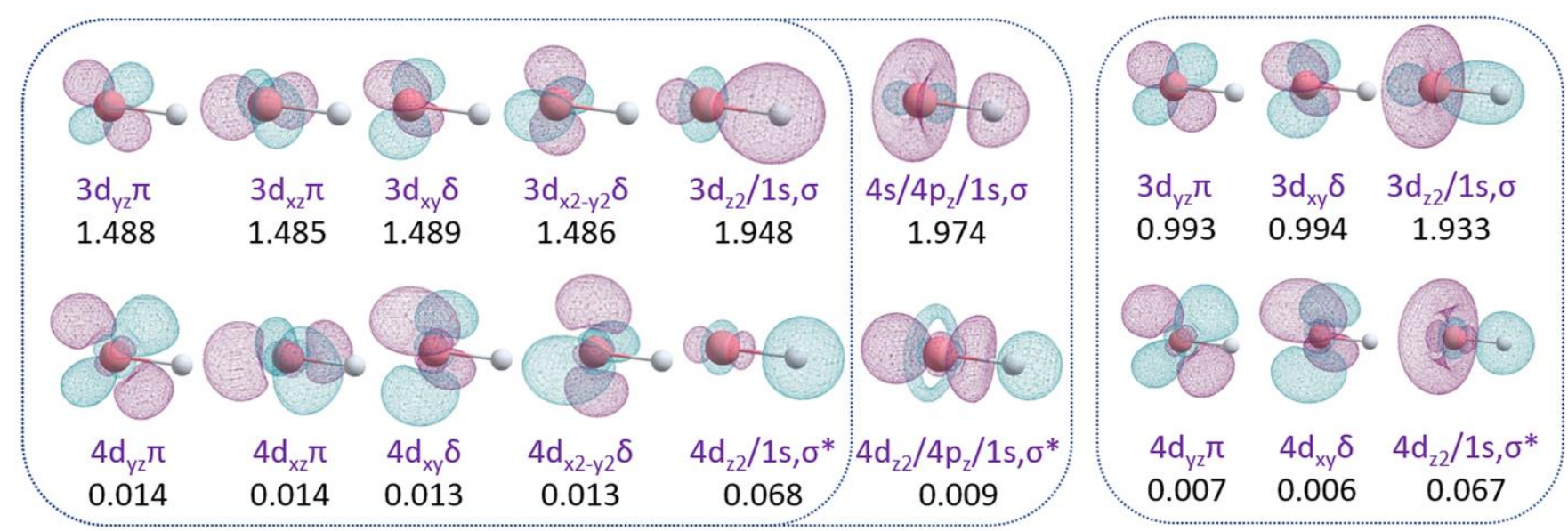

Figure 4. $\mathrm{CoH}$ natural orbitals and corresponding occupation numbers in the ext-CPO (10e,12o) (left) and nom-CPO (4e6o) (right) active spaces. The outlined region of ext-CPO space includes orbitals of the mod-CPO (8e10o) active space.

The equilibrium dipole moments computed with nom-CPO and mod-CPO active spaces using the def2-TZVPD basis set are summarized in Table 3. The ground state geometries were optimized using analytical (CASSCF, tPBE, tOPBE, CASPT2) and numerical (MRCISD) gradients and then the corresponding dipole moments were calculated analytically by CASSCF, tPBE, tOPBE, and CASPT2 and numerically by MRCISD+Q. The tPBE0 dipole moments were computed analytically using tPBE geometries. The deviations of equilibrium bond lengths from the experimental values are small for all molecules with MUEs of $0.03 \AA$ (CASSCF and CASPT2), $0.02 \AA$ (MRCISD+Q), and $0.01 \AA$ (tPBE) (see Table S3 in SI). These small errors in the optimized geometries on average result in $\sim 0.1 \mathrm{D}$ difference between the equilibrium and single-point values computed at the experimental internuclear distances (Table 3 and Table S4).

Table 3. Experimental and computed equilibrium dipole moments (in debyes) found with CASSCF, MCPDFT, CASPT2, and MRCISD+Q using the def2-TZVPD basis set. The weights of the leading configurations and the multireference $\mathrm{M}$ diagnostics are found with the mod-CPO active space. All calculations are at the fixed experimental distances $r^{\text {Exptl }}(\AA)$.

\begin{tabular}{|c|c|c|c|c|c|c|c|c|c|c|c|c|c|c|}
\hline \multirow{2}{*}{ Species } & \multirow{2}{*}{$r^{\text {Exptl }}$} & \multirow{2}{*}{$\mu^{\text {Exptl }}$} & \multicolumn{6}{|c|}{$\mu$, nom-CPO } & \multicolumn{6}{|c|}{$\mu$, mod-CPO } \\
\hline & & & CASSCF & tOPBE & $\mathrm{tPBE}$ & tPBE0 & CASPT2 & MRCISD+Q & CASSCF & tOPBE & E tPBE & tPBE0 & CASPT2 & 2 MRCISD+Q \\
\hline $\mathrm{CoH}\left({ }^{3} \Phi\right)$ & 1.514 & $2.63^{\mathrm{a}}$ & 3.32 & - & - & - & 3.01 & 2.29 & 4.11 & 2.73 & 2.41 & 2.84 & 3.20 & 2.86 \\
\hline $\mathrm{CrH}\left({ }^{6} \Sigma^{+}\right)$ & 1.655 & $3.501(33)$ & 4.09 & 3.21 & 2.63 & 3.00 & 3.79 & 3.83 & 4.09 & 3.21 & 2.63 & 3.00 & 3.79 & 3.83 \\
\hline $\mathrm{CrN}\left({ }^{4} \Sigma^{-}\right)$ & 1.565 & $2.31(4)$ & 2.08 & 2.77 & 3.18 & 2.90 & - & 2.69 & 2.08 & 2.77 & 3.18 & 2.90 & - & 2.69 \\
\hline $\mathrm{CrO}\left({ }^{5} \Pi\right)$ & 1.615 & $3.88(13)$ & 3.36 & 3.88 & 3.90 & 3.77 & 3.80 & 3.79 & 3.36 & 3.88 & 3.90 & 3.77 & 3.80 & 3.79 \\
\hline
\end{tabular}




\begin{tabular}{|c|c|c|c|c|c|c|c|c|c|c|c|c|c|}
\hline $\operatorname{FeC}\left({ }^{3} \Delta\right)$ & $1.5892 .36(3)$ & 1.68 & 2.52 & 2.78 & 2.51 & 2.29 & 2.16 & 1.63 & 2.64 & 2.83 & 2.53 & 2.27 & 2.15 \\
\hline $\mathrm{FeH}\left({ }^{4} \Delta\right)$ & $1.6062 .63(3)$ & - & - & - & - & - & - & 4.25 & 2.73 & 2.43 & 2.88 & 3.07 & 2.60 \\
\hline $\mathrm{NiH}\left({ }^{2} \Delta\right)$ & $1.4542 .44(2)$ & 2.78 & 4.94 & 4.18 & 3.83 & 4.33 & 2.68 & 3.88 & 2.61 & 2.28 & 2.68 & 3.07 & 2.81 \\
\hline $\operatorname{ScF}\left({ }^{1} \Sigma^{+}\right)$ & $1.7871 .72(2)$ & 1.78 & 1.78 & 2.26 & 2.14 & 1.79 & 1.71 & 1.51 & 1.59 & 2.07 & 1.93 & 1.78 & 1.60 \\
\hline $\mathrm{ScH}\left({ }^{1} \Sigma^{+}\right)$ & $1.7751 .74(15)$ & 1.10 & 1.73 & 1.96 & 1.74 & 1.53 & 1.32 & 1.17 & 1.73 & 1.87 & 1.70 & 1.30 & 1.25 \\
\hline $\mathrm{ScO}\left({ }^{2} \Sigma^{+}\right)$ & $1.6663 .67^{b}$ & 3.27 & 2.89 & 3.69 & 3.58 & 3.70 & 3.79 & 3.27 & 2.89 & 3.69 & 3.58 & 3.70 & 3.79 \\
\hline $\operatorname{ScS}\left({ }^{2} \Sigma^{+}\right)$ & $2.1385 .64(4)$ & 4.58 & 4.35 & 5.23 & 5.07 & 5.23 & 5.23 & 4.58 & 4.35 & 5.23 & 5.07 & 5.23 & 5.23 \\
\hline $\operatorname{TiH}\left({ }^{4} \Phi\right)$ & $1.7772 .455(6)$ & 1.95 & 2.49 & 3.03 & 2.76 & 2.18 & 2.28 & 2.00 & 2.62 & 3.16 & 2.87 & 2.26 & 2.34 \\
\hline $\operatorname{TiN}\left({ }^{2} \Sigma^{+}\right)$ & $1.5803 .56(5)$ & 2.67 & 2.85 & 3.57 & 3.35 & 3.39 & 3.25 & 2.67 & 2.85 & 3.57 & 3.35 & 3.39 & 3.25 \\
\hline $\mathrm{TiO}\left({ }^{3} \Delta\right)$ & $1.6203 .34(1)$ & 2.80 & 2.63 & 3.41 & 3.26 & 3.19 & 3.32 & 2.80 & 2.63 & 3.41 & 3.26 & 3.19 & 3.32 \\
\hline $\operatorname{TiS}\left({ }^{3} \Delta\right)$ & $2.0835 .75(10)$ & 4.47 & 5.31 & 5.69 & 5.39 & 5.26 & 5.31 & 4.47 & 5.31 & 5.69 & 5.39 & 5.26 & 5.31 \\
\hline $\mathrm{VN}\left({ }^{3} \Delta\right)$ & $1.5703 .07(7)$ & 2.31 & 2.68 & 3.26 & 3.02 & 2.91 & 2.87 & 2.31 & 2.68 & 3.26 & 3.02 & 2.91 & 2.87 \\
\hline $\operatorname{VO}\left({ }^{4} \Sigma^{-}\right)$ & $1.5893 .355(5)$ & 2.58 & 2.96 & 3.50 & 3.27 & 3.07 & 3.22 & 2.58 & 2.96 & 3.50 & 3.27 & 3.07 & 3.22 \\
\hline $\operatorname{VS}\left({ }^{4} \Sigma^{-}\right)$ & $2.0535 .16(5)$ & 5.57 & 5.73 & 4.79 & 4.98 & 5.43 & 5.66 & 5.57 & 5.73 & 4.79 & 4.98 & 5.43 & 5.66 \\
\hline MSE & & -0.36 & -0.08 & 0.19 & 0.04 & 0.04 & -0.07 & -0.16 & -0.18 & 0.04 & -0.01 & -0.01 & -0.04 \\
\hline MUE & & 0.61 & 0.55 & 0.41 & 0.32 & 0.33 & 0.25 & 0.77 & 0.39 & 0.29 & 0.24 & 0.28 & 0.25 \\
\hline
\end{tabular}

$\overline{\mathrm{a}}(18 \mathrm{e}, 14 \mathrm{o}) \mathrm{MRCISD}+\mathrm{Q} / 5 \zeta\left(\mathrm{ref}^{105}\right) ;{ }^{\mathrm{b}}(7 \mathrm{e} 12 \mathrm{o})$ MRCISD+Q/aug-cc-pVQZ+DKH2 $\left(\right.$ ref $\left.^{102}\right) ;{ }^{\mathrm{c}}(8 \mathrm{e}, 9 \mathrm{o})$ active space

In general, tOPBE, tPBE, tPBE0, CASPT2, and MRCISD+Q methods all improve upon CASSCF as can be seen from the mean unsigned errors with respect to the reference values (MUE) (Table 3). We use experimental values as references for all molecules, except for $\mathrm{CoH}$ and $\mathrm{ScO}$, where the references are the theoretical estimates of equilibrium dipole moments as discussed above. The MUE of 0.61 D (CASSSCF) in the nom-CPO reduces to $0.55 \mathrm{D}$ (tOPBE), $0.41 \mathrm{D}$ (tPBE), $0.32 \mathrm{D}$ (tPBE0), $0.33 \mathrm{D}$ (CASPT2), and 0.25 D (MRCISD+Q). Similarly, the MUE of 0.77 D (CASSSCF) in the mod-CPO reduces to $0.39 \mathrm{D}$ (tOPBE), 0.29 $\mathrm{D}$ (tPBE), 0.24 $\mathrm{D}$ (tPBE0), 0.28 D (CASPT2), and 0.25 D (MRCISD+Q). These results are encouraging since the computational cost of MC-PDFT is much lower than that of CASPT2 or MRCISD+Q.

Note that $\mathrm{FeH}$ was excluded from averaging in the nom-CPO case because of the instability of the $(5 e, 80)$ active space, which is subject to severe orbital rotations. These rotations exchange the desired $\sigma^{*}\left(d_{z^{2}} / 1 s\right)$ orbital with the competing $\sigma^{*}\left(p_{z} / 1 s\right)$ orbital and make the active space inconsistent during geometry optimization (Figure S3). At the internuclear distance of $1.606 \AA$, the $\operatorname{PBE}($ nom-CPO) dipole moment of $2.84 \mathrm{D}$ is in good agreement with the experimental value of 2.63(3) $\mathrm{D}$, whereas the unbalanced $(5 \mathrm{e}, 8 \mathrm{o})$ active space characterized by $\sigma^{*}\left(d_{z^{2}} / 1 s\right) \rightarrow \sigma^{*}\left(p_{z} / 1 s\right)$ rotation yields a dramatically large value 
of $9.82 \mathrm{D}$. This is an unphysical result originating from a wrong active space. In the nom-CPO, the occupation numbers of $\sigma\left(d_{z^{2}} / 1 s\right)$ and $\sigma^{*}\left(d_{z^{2}} / 1 s\right)$ orbitals are 1.88 and 0.12 , respectively. In contrast, in the unbalanced $(5 \mathrm{e}, 80)$ active space the respective occupations of $\sigma\left(d_{z^{2}} / 1 s\right)$ and $\sigma^{*}\left(p_{z} / 1 s\right)$ are 1.45 and 0.56. Unlike the nom-CPO case, the mod-CPO active space shows much greater stability during geometry optimization and results in a $2.43 \mathrm{D}$ equilibrium dipole moment, which is only $0.2 \mathrm{D}$ smaller than the reference value. A large $4.18 \mathrm{D}$ deviation in $\mathrm{tPBE}($ nom-CPO) from the experimental benchmark of 2.44(2) is also observed in $\mathrm{NiH}$. However, this discrepancy is greatly eliminated at the mod-CPO level, which predicts a value of 2.28 D. The overall improvement of the dipole moments upon expansion of the active space from nom-CPO to mod-CPO together with increasing stability of the active space suggests that the mod-CPO should be preferred over the nom-CPO scheme, as one would expect.

The ext-CPO scheme appears to be less balanced than mod-CPO at least in the case of the metal sulfides due to the rotation of the $3 \mathrm{~d}$ orbitals of the sulfur atom into the active space. For example, the mod-CPO active space in vanadium sulfide, VS, consists of the nine valence electrons distributed in twelve molecular orbitals. The ground state has ${ }^{4} \Sigma^{-}$symmetry and two out of three singly occupied orbitals $3 d_{x y}$ and $3 d_{x^{2}-y^{2}}$, are correlated to the $4 d_{x y}$ and $4 d_{x^{2}-y^{2}}$ orbitals, as illustrated in Figure 5 , left. This balanced active space leads to the equilibrium dipole moments of 4.74 D (tPBE), 5.32 D (CASPT2), and 5.66 D (MRCISD+Q) compared to the experimental benchmark of 5.16(5) D. In contrast, rotation of $3 d_{x y}(\mathrm{~S})$ and $3 d_{x^{2}-y^{2}}(\mathrm{~S})$ orbitals mainly localized on the $\mathrm{S}$ atom into the active space results in the lowerenergy sulfur-polarized active space with the erratic tPBE value of $0.11 \mathrm{D}$ (Figure 5, center). This dramatically wrong tPBE value can be attributed to the fact that the active space becomes unbalanced as there is no correlation between singly occupied orbitals and $3 d_{x y}(\mathrm{~S})$ and $3 d_{x^{2}-y^{2}}(\mathrm{~S})$ orbitals. It is interesting that the 0.02 occupation numbers of each of these two orbitals are even larger than occupation numbers of $4 d_{x y}$ and $4 d_{x^{2}-y^{2}}$ orbitals in the balanced mod-CPO. This population arises from the correlation of bonding $\sigma$ and $\pi$ orbitals to the $3 d_{x y}(\mathrm{~S})$ and $3 d_{x^{2}-y^{2}}(\mathrm{~S})$ in the unbalanced (9e12o) active space. The expansion of the active space by including the lone-pair occupied $3 \mathrm{~s}(\mathrm{~S})$ and correlated unoccupied $4 \mathrm{~s}(\mathrm{~S})$ orbitals facilitates correlation of the bonding orbitals to $3 d_{x y}(\mathrm{~S})$ and $3 d_{x^{2}-y^{2}}(\mathrm{~S})$ orbitals instead of $3 d_{x y}(\mathrm{~V})$ and $3 d_{x^{2}-y^{2}}(\mathrm{~V})$ (Figure 5, right). This leads to $1.97 \mathrm{D}$ (tPBE) and $3.64 \mathrm{D}(\mathrm{CASPT})$ dipole moments at the ext-CPO level, which deviate significantly from the experimental 5.16(5) D. 


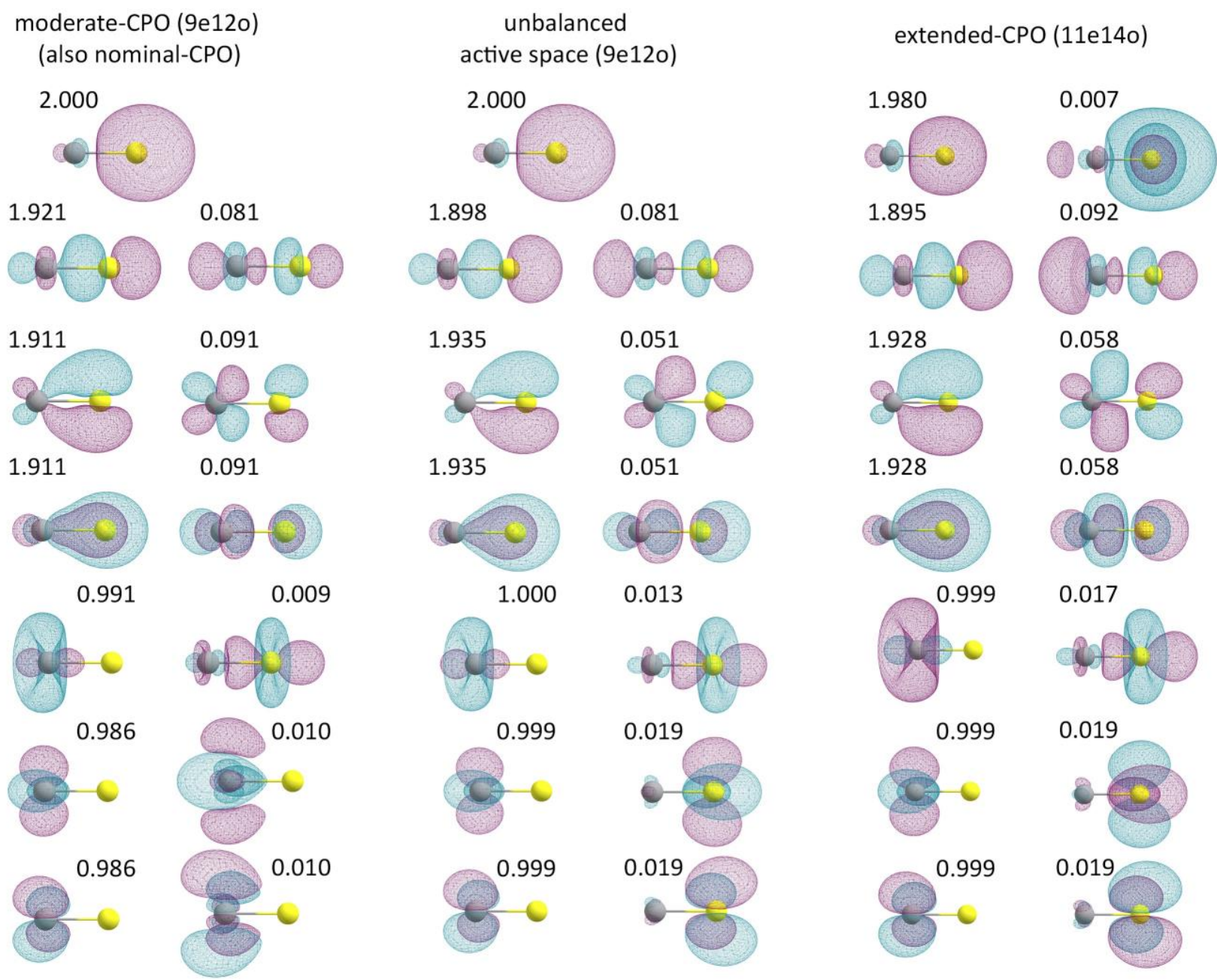

Figure 5. VS natural orbitals and occupation numbers for the mod-CPO (9e12o) (= nom-CPO), unbalanced (9e12o) (see text), and ext-CPO (11e,14o) active spaces. The doubly occupied 3s(S) orbitals are shown for completeness only and not included in the (9e12o) active space.

In a similar way, the rotation of $3 \mathrm{~d}(\mathrm{~S})$ orbital in the active space prevents formation of the desired mod-CPO active space in TiS. The unbalanced sulfur-polarized active space largely underestimates the experimental reference of 5.75(10) D with the tPBE value of only 4.88 D. However, considering the low occupation of the $3 \mathrm{~d}^{\prime}(\mathrm{Ti})$ orbital in the mod-CPO active space (That is expected due to the correlation of $3 d^{\prime}(\mathrm{Ti})$ to the singly occupied $3 \mathrm{~d}(\mathrm{Ti})$ orbital), we attempted to eliminate this orbital from the active space, reducing its size to $(8 \mathrm{e}, 9 \mathrm{o})$ from the original $(8 \mathrm{e}, 10 \mathrm{o})$ in mod-CPO. This allowed us to avoid undesired rotations and obtain a closer agreement between the $\operatorname{tPBE}(8 \mathrm{e}, 9 \mathrm{o})$ prediction $(5.69 \mathrm{D})$ and the measured value $(5.75(10) \mathrm{D})$. 
The signed errors in equilibrium dipole moments obtained with the mod-CPO scheme are shown in Figure 6 where positive errors mean that the molecule is more polar than the reference and negative errors that it is less polar. It is not surprising that the largest deviations up to $1.6 \mathrm{D}$ are found with the CASSCF method, which is missing a significant part of the dynamic correlation. The error is largely reduced by tPBE in most cases, with the exceptions being $\mathrm{CrH}, \mathrm{CrN}, \mathrm{TiH}$, and VS. While the first three molecules are indeed problematic for tPBE with the errors being as large as $0.9 \mathrm{D}$, the tPBE error in VS is still comparable to the other methods. Note that according to eq (15), the MC-PDFT dipole is a sum of the parent CASSCF dipole moment and a correction that depends on the electron density and the on-top pair density. In general, the tPBE correction to the CASSCF tends to reverse the sign of the error increasing the polarity when it is underestimated by CASSCF and decreasing the polarity when it is overestimated by CASSCF. This accounts for the success of tPBE0 because it scales down the correction term by a factor of $\lambda$, making predictions closer to the reference values. The opposite trends in CASSCF and MC-PDFT errors are responsible for the improvement of tPBE0 results for all molecules except ScS, where the CASSCF and tPBE errors have the same sign and where the tPBE0 is expected to have a larger error with respect to tPBE. However, for $\mathrm{ScS}$ all methods predict a lower value than the reference; therefore, tPBE0 results are expected to be close to MRCISD+Q and CASPT2. 

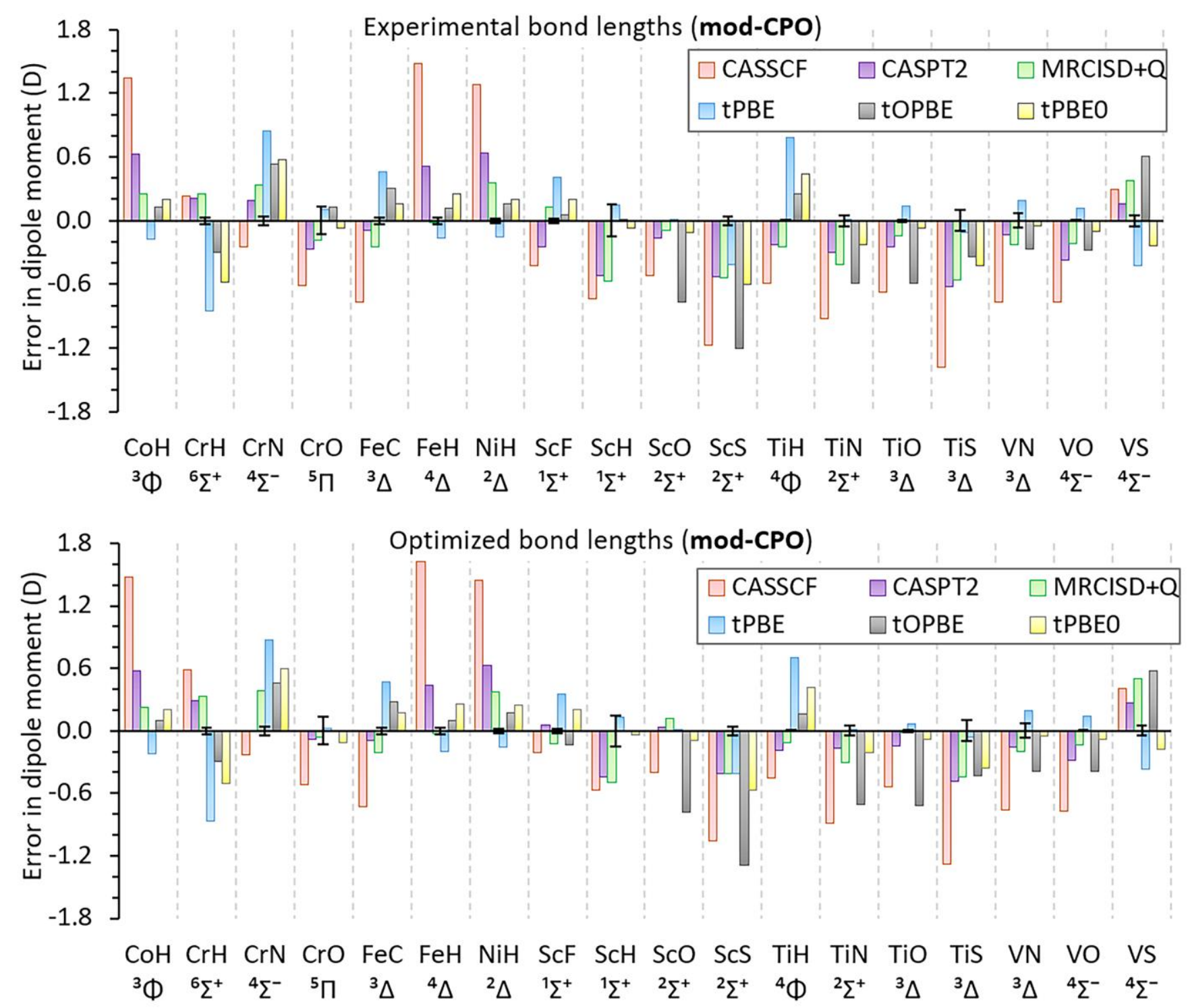

Figure 6. Deviations between the dipole moments computed with mod-CPO active spaces from the reference values. The internuclear distances are fixed to experimental ones (top) and optimized (bottom). The references are experimental dipole moments, except for $\mathrm{CoH}$ and $\mathrm{ScO}$ represented by the best theoretical estimates. The error bars indicate uncertainties in experimental values. In the case of TiS, the mod-CPO is reduced to $(8 \mathrm{e}, 9 \mathrm{o})$ active space.

\section{A Detailed Look at CrH}

The problematic species, $\mathrm{CrH}(M=0.09), \mathrm{CrN}(M=0.16)$, and $\mathrm{TiH}(M=0.78)$ differ in their bonding type and symmetry and in the multiconfigurational character of the ground-state wave function. The simplest problematic molecule is chromium hydride, which has a single bond and is in the highest spin state, $S=5 / 2$. The reference dipole moment of $\mathrm{CrH}, 3.50(3) \mathrm{D}$, is in good agreement with a value 
previously calculated from an MRCISD density and is likely to be correct. ${ }^{101}$ The ground and excited states of $\mathrm{CrH}$ are well-separated near equilibrium, but at the $\mathrm{Cr}\left({ }^{6} \mathrm{~S}\right)+\mathrm{H}\left({ }^{2} \mathrm{~S}\right)$ dissociation limit, the ground ${ }^{6} \Sigma^{+}$state becomes degenerate with the excited ${ }^{8} \Sigma^{+}$state. ${ }^{114}$ The possible reasons behind the large error in $\mathrm{CrH}$ dipole moment include but are not limited to: (i) quality of the active space (ii) quality of the basis set (iii) translation scheme used to obtain the on-top functional (iv) lack of nonlocal exchange in the tPBE on-top functional; and (v) type of the parent density functional. Next we explore each of these possibilities.

The mod-CPO active space already embraces all the valence electrons, and it is equivalent to the nom-CPO and ext-CPO schemes for this molecule; however, the active space can be expanded by adding unoccupied $5 s$ and $4 p$ subshells, as was suggested in the previous CASPT2 ${ }^{115}$ and MRCISD ${ }^{114}$ studies of $\mathrm{CrH}$. The active space dependence of the tPBE dipole moment curves is shown in the left panels of Figure 7. In general, the tPBE results are more sensitive to the choice of the active space than are the CASSCF ones. In Figure 7, (7e,12o) refers to the mod-CPO active space, and (7e,13o) is built upon (7e,12o) by adding a single $a_{1}$ orbital. The addition of an extra $a_{1}$ orbital has only a small effect on the DMC at most geometries (although the DMC is slightly smoother near the equilibrium geometry), but it does lead to a pronounced (apparently unphysical) spike between 2.6 and $3.0 \AA$. This spike arises from the orbital-space rotations of weakly correlated $a_{1}$ orbitals during the self-consistency iterations that make the active space inconsistent in this region as illustrated in Figure S7. The expansion of the active space to $(7 \mathrm{e}, 16 \mathrm{o})$ by adding two $a_{1}$, one $b_{1}$, and one $b_{2}$ orbitals $(5 s(\mathrm{Cr})$ - and $4 p(\mathrm{Cr})$-shells) to the $(7 \mathrm{e}, 12 \mathrm{o})$ space does not resolve the problem completely because there is a small irregularity in the $\operatorname{PBE}(7 \mathrm{e}, 160)$ curve around $3.0 \AA$ owing to different CASSCF solutions (Figure S7, right). However, the (7e,17o) active space obtained by adding three $a_{1}$, one $b_{1}$, and one $b_{2}$ orbital $(5 s(\mathrm{Cr}), 4 p(\mathrm{Cr})$, and $2 s(\mathrm{H})$ subshells $)$ to the $(7 \mathrm{e}, 12 \mathrm{o})$ active space prevents the orbital rotation and makes the tPBE dipole moment curve a smooth function of the internuclear distance out to $3.6 \AA$. At larger distances, the rotation of the $4 f$ orbitals corrupts the consistency of the active space as the occupations of weakly correlated orbitals become small. The rightside panels of Figure 7 show that the potential energy curve improves upon the active space expansion from $(7 \mathrm{e}, 12 \mathrm{o})$ to $(7 \mathrm{e}, 17 \mathrm{o})$ for all considered on-top functionals. Taking $3.6 \AA$ as the dissociation limit, the equilibrium dissociation energy obtained with $\operatorname{tPBE}(7 \mathrm{e}, 17 \mathrm{o}) / \mathrm{def} 2-\mathrm{TZVPD}$ would $47.6 \mathrm{kcal} / \mathrm{mol}$, in good agreement with the experimental value ${ }^{116}$ of $46.8(2) \mathrm{kcal} / \mathrm{mol}$; however, the last panel of Figure 7 shows that the actual dissociation energy of $\mathrm{CrH}$ would be slightly larger than the value calculated at $3.6 \AA$. Despite the improvement of the MC-PDFT equilibrium dissociation energy, there is little improvement in the equilibrium dipole moment of $\mathrm{CrH}$ with the expansion of the active space. 

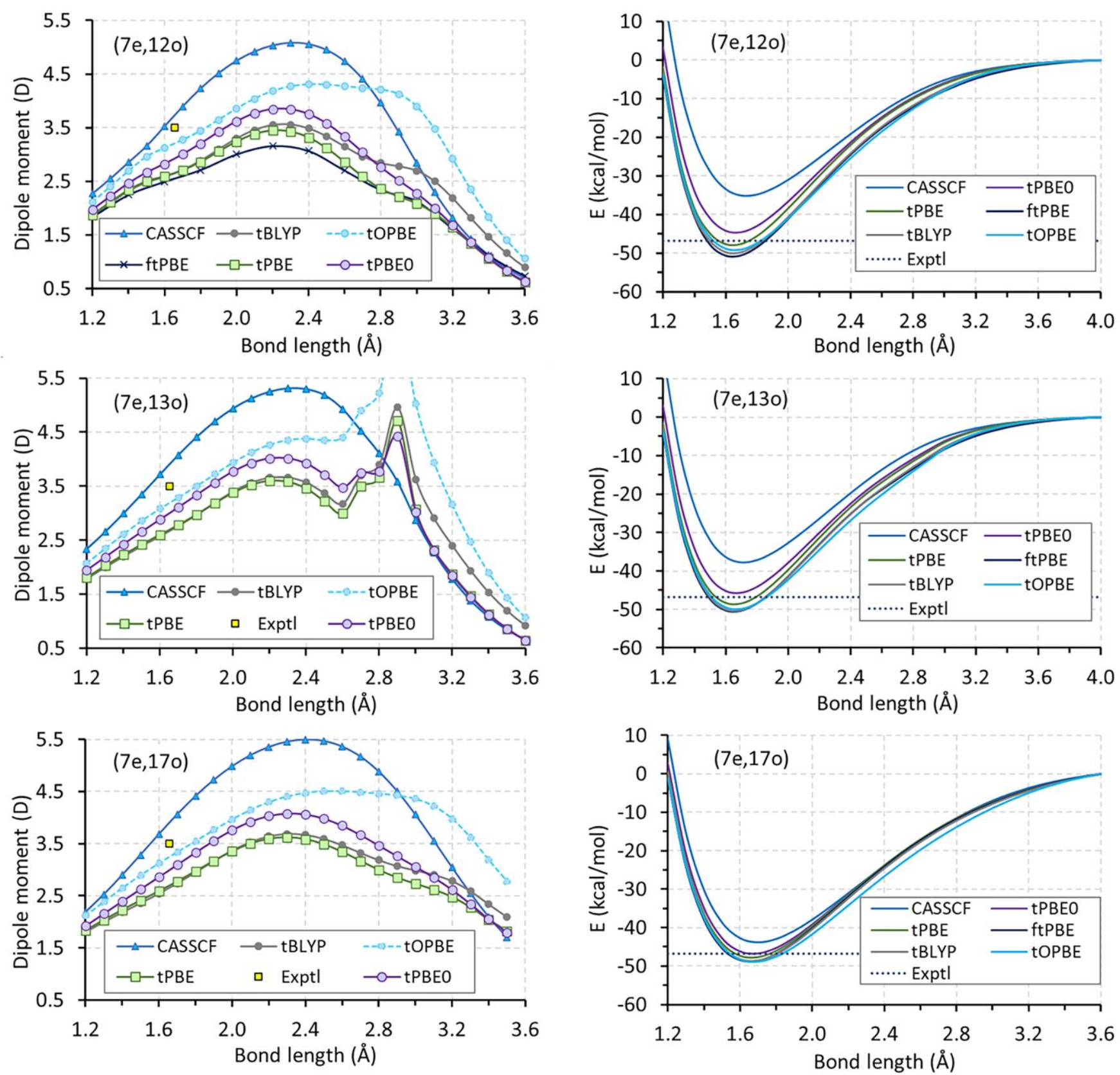

Figure 7. The active space dependence of the dipole moments (left) and potential energy curves (right) of the $X^{6} \Sigma^{+}$state in $\mathrm{CrH}$. All dipole moments are computed analytically except for numerical ftPBE results. All methods share the same parent CASSCF wave function for a given geometry. A yellow square indicates the experimental dipole moment (left) and a dotted line shows the dissociation energy (right).

Next, we consider the effect of increasing the basis set on the $\mathrm{CrH}$ results. The increase of the basis set from def2-TZVPD to def2-TZVPPD by adding $d$ functions to $\mathrm{H}$ and $g$ functions to $\mathrm{Cr}$ has a minor effect on the $\operatorname{tPBE}(7 \mathrm{e}, 12 \mathrm{o})$ dipole moment, which changes from 2.65 to $2.63 \mathrm{D}$ at the fixed experimental 
equilibrium distance of $1.655 \AA$. An even larger basis set, def2-QZVPPD, also does not significantly affect the dipole moment; it yields $2.61 \mathrm{D}$. This is consistent with a previous density functional study ${ }^{56}$ where the def2-TZVPD and def2-TZVPPD basis sets were shown to have small errors when comparing DFT dipole moments with benchmark $\operatorname{CCSD}(\mathrm{T})$ values. Thus, the basis set is not responsible for the observed inaccuracy of the $\mathrm{CrH}$ dipole moment.

The translation scheme used in PBE has the on-top energy depending on the electron density, its gradient, and the on-top pair density. Another translation scheme, known as full translation (ft), adds a dependence on the gradient of the on-top pair density. This dependence complicates the analytical expression of the dipole moment; therefore, we evaluated the ftPBE dipole moments numerically. The ftPBE $(7 e, 120)$ results shown in Figure 7 show that the equilibrium dipole moment is slightly worse with ftPBE than with tPBE.

The finding that all the problematic molecules have a high spin with multiple unpaired electron suggests that perhaps the exchange part of the functional underlying the translation might be responsible for the observed deviations. The hybrid tPBE0 is only partly successful in reducing the error in the $\mathrm{CrH}$ dipole moment; it reduces it because CASSCF overestimates and tPBE underestimates the reference values, but tPBE0 does not remove the irregularity in the (7e,13o) DMC, and it does not give good agreement with the experimental dipole moment for any of the active spaces.

Finally, we explore the type of the parent density functional. The translated BLYP functional that has Becke exchange and LYP correlation yields virtually the same results as the translated PBE, which has PBE exchange and PBE correlation (Figure 7). On the other hand, replacement of the PBE exchange part with the OPTX exchange functional ${ }^{117}$ largely reduces the error in the $\mathrm{CrH}$ equilibrium dipole from -0.85 to $-0.29 \mathrm{D}$ in the corresponding tOPBE on-top functional. However, the overall performance of tOPBE $(\mathrm{MUE}=0.39 \mathrm{D})$ on the whole set of diatomic molecules is worse than tPBE (MUE $=0.29 \mathrm{D}$ ) and tPBE0 (MUE $=0.24 \mathrm{D}$ ) due to unsatisfactory results for the medium-spin molecules. In the pathological case of ScS, the tOPBE error is as large as 1.3 D. Therefore, we do not recommend the tOPBE functional as the best functional overall. Instead, among the functionals studied here, the tPBE0 is expected to be the most reliable functional for dipole moment evaluations. We speculate that a machinelearned functional, ${ }^{118}$ if parametrized using dipole moments, would be able to improve the dipole moments over tPBE0. 


\section{CONCLUDING REMARKS}

In this work, we presented the MC-PDFT and HMC-PDFT analytical expressions for the electric dipole moment. We showed that the MC-PDFT and HMC-PDFT dipole moments can be written as the sum of CASSCF dipole moment and a correction depending on the on-top density functional that depends on the density and the on-top pair density. The MC-PDFT and HMC-PDFT analytical dipole moments are implemented in the $m r h$ add-on of the $P y S C F$ package, and we used this implementation to investigate their performance for predicting equilibrium dipole moments of the multireference systems and the dipole moments of single-reference systems in nonequilibrium regions where the wave functions acquire significant multiconfigurational character and cannot be well described by a single Slater determinant. We examined three on-top functionals (tPBE, ftPBE, tOPBE) for MC-PDFT and one (tPBE0) for HMCPDFT.

We showed that MC-PDFT dipole moment curves have correct asymptotic behaviors for homolytically dissociating species and significantly improve upon the CASSCF results by capturing more electron correlation. The dipole moment curves of $\mathrm{HF}, \mathrm{CO}, \mathrm{NO}$, and $\mathrm{AlO}$ species are well reproduced by tPBE in the vicinity of the equilibrium and close to the dissociation limit, but the magnitude of the dipole moment is overestimated at the inflection points of the dipole moment functions. We showed that HMCPDFT with tPBE0 on-top functional is more accurate than MC-PDFT/tPBE for both equilibrium and nonequilibrium dipole moments. Usually, MC-PDFT (tPBE) overcorrects the CASSCF dipole moments, and the success of tPBE0, which represents the total energy as the weighted average of CASSCF and MCPDFT energies, can be ascribed to the reduced on-top density correction to the CASSCF dipole moments that reverses the sign of the error and reduces its magnitude. The improvement achieved by tPBE0 comes with no additional computational cost compared to tPBE.

We also showed that the moderate CPO scheme for selecting orbitals in the active space is more accurate and robust than the nom-CPO scheme. We found that the mean unsigned deviations of the equilibrium dipole moments from the reference values of 18 first-row transition metal diatomics predicted by MC-PDFT with mod-CPO are $0.29 \mathrm{D}$ for $\mathrm{tPBE}$ and $0.24 \mathrm{D}$ for tPBE0. These MUEs are comparable to the errors obtained with the CASPT2 $(0.28 \mathrm{D})$ and MRCISD+Q $(0.25 \mathrm{D})$ methods. Overall, these results are encouraging because MC-PDFT is much more affordable than the CASPT2 and MRCISD+Q methods. 


\section{ASSOCIATED CONTENT}

Supporting information includes numerical and analytical dipole moments of $\mathrm{CHFClBr}$, optimized bond lengths, dipole moments of first-row transition metal diatomics computed at the fixed experimental distances, and natural molecular orbitals.

\section{AUTHOR INFORMATION}

Aleksandr O. Lykhin, Department of Chemistry, Pritzker School of Molecular Engineering, The James Franck Institute and Chicago Center for Theoretical Chemistry, The University of Chicago, Chicago, IL 60637, United States

orcid.org/0000-0002-9366-5866

Email: 1ykhin@uchucago.edu

Donald G. Truhlar, Department of Chemistry, Chemical Theory Center, and Minnesota Supercomputing Institute, University of Minnesota, 207 Pleasant Street SE, Minneapolis, Minnesota 55455, United States orcid.org/0000-0002-7742-7294

Email: truhlar@umn.edu

Laura Gagliardi, Department of Chemistry, Pritzker School of Molecular Engineering, The James Franck Institute and Chicago Center for Theoretical Chemistry, The University of Chicago, Chicago, IL 60637, United States; Argonne National Laboratory, Lemont, Illinois 60439, United States orcid.org/0000-0001-5227-1396

Email: 1gagliardi@uchicago.edu

\section{Notes}

The authors declare no competing financial interest. 


\section{ACKNOWLEDGMENTS}

This work was supported by the Air Force Office of Scientific Research by Grant No. FA9550-16-1-0134. We thank the University of Chicago Research Computing Center for computational resources.

\section{REFERENCES}

(1) Stone, A. J. The Theory of Intermolecular Forces; Oxford University Press: Oxford, UK, 2013.

(2) Puzzarini, C. Rotational Spectroscopy Meets Theory. Phys. Chem. Chem. Phys. 2013, 15 (18), 6595-6607. https://doi.org/10.1039/c3cp44301a.

(3) Brown, J.; Carrington, A. Rotational Spectroscopy of Diatomic Molecules; Cambridge University Press: Cambridge, UK, 2003.

(4) Gdanitz, R. J.; Ahlrichs, R. The Averaged Coupled-Pair Functional (ACPF): A Size-Extensive Modification of MRCI(SD). Chem. Phys. Lett. 1988, 143 (5), 413-420. https://doi.org/10.1016/0009-2614(88)87388-3.

(5) Würthner, F. Dipole-Dipole Interaction Driven Self-Assembly of Merocyanine Dyes: From Dimers to Nanoscale Objects and Supramolecular Materials. Acc. Chem. Res. 2016, 49 (5), 868-876. https://doi.org/10.1021/acs.accounts.6b00042.

(6) Schwartz, J. J.; Mendoza, A. M.; Wattanatorn, N.; Zhao, Y.; Nguyen, V. T.; Spokoyny, A. M.; Mirkin, C. A.; Baše, T.; Weiss, P. S. Surface Dipole Control of Liquid Crystal Alignment. J. Am. Chem. Soc. 2016, 138 (18), 5957-5967. https://doi.org/10.1021/jacs.6b02026.

(7) Tscherbul, T. V.; Krems, R. V. Tuning Bimolecular Chemical Reactions by Electric Fields. Phys. Rev. Lett. 2015, 115 (2), 1-5. https://doi.org/10.1103/PhysRevLett.115.023201.

(8) Krems, R. V. Molecules near Absolute Zero and External Field Control of Atomic and Molecular Dynamics. Int. Rev. Phys. Chem. 2005, 24 (1), 99-118. https://doi.org/10.1080/01442350500167161.

(9) Faber, F. A.; Hutchison, L.; Huang, B.; Gilmer, J.; Schoenholz, S. S.; Dahl, G. E.; Vinyals, O.; Kearnes, S.; Riley, P. F.; Von Lilienfeld, O. A. Prediction Errors of Molecular Machine Learning Models Lower than Hybrid DFT Error. J. Chem. Theory Comput. 2017, 13 (11), 5255-5264. https://doi.org/10.1021/acs.jctc.7b00577.

(10) Chen, C.; Ye, W.; Zuo, Y.; Zheng, C.; Ong, S. P. Graph Networks as a Universal Machine Learning Framework for Molecules and Crystals. Chem. Mater. 2019, 31 (9), 3564-3572.

https://doi.org/10.1021/acs.chemmater.9b01294.

(11) Nebgen, B.; Lubbers, N.; Smith, J. S.; Sifain, A. E.; Lokhov, A.; Isayev, O.; Roitberg, A. E.; Barros, K.; Tretiak, S. Transferable Dynamic Molecular Charge Assignment Using Deep Neural Networks. J. Chem. Theory Comput. 2018, 14 (9), 4687-4698. https://doi.org/10.1021/acs.jctc.8b00524.

(12) Wiberg, K. B.; Rablen, P. R. Atomic Charges. J. Org. Chem. 2018, 83 (24), 15463-15469. https://doi.org/10.1021/acs.joc.8b02740.

(13) Cramer, C. J.; Truhlar, D. G. A Universal Approach to Solvation Modeling. Acc. Chem. Res. 2008, 41 (6), 
760-768. https://doi.org/10.1021/ar800019z.

(14) Bleiziffer, P.; Schaller, K.; Riniker, S. Machine Learning of Partial Charges Derived from High-Quality Quantum-Mechanical Calculations. J. Chem. Inf. Model. 2018, 58 (3), 579-590.

https://doi.org/10.1021/acs.jcim.7b00663.

(15) Neugebauer, J.; Reiher, M.; Kind, C.; Hess, B. A. Quantum Chemical Calculation of Vibrational Spectra of Large Molecules - Raman and IR Spectra for Buckminsterfullerene. J. Comput. Chem. 2002, 23 (9), 895910. https://doi.org/10.1002/jcc.10089.

(16) Long, D. A. The Raman Effect: A Unified Treatment of the Theory of Raman Scattering by Molecules. 2002; 2002; Vol. 8.

(17) Fedorov, D. A.; Derevianko, A.; Varganov, S. A. Accurate Potential Energy, Dipole Moment Curves, and Lifetimes of Vibrational States of Heteronuclear Alkali Dimers. J. Chem. Phys. 2014, 140 (18), 184315. https://doi.org/10.1063/1.4875038.

(18) Nikitin, A. V.; Rey, M.; Tyuterev, V. G. High Order Dipole Moment Surfaces of $\mathrm{PH}_{3}$ and Ab Initio Intensity Predictions in the Octad Range. J. Mol. Spectrosc. 2014, 305, 40-47. https://doi.org/10.1016/j.jms.2014.09.010.

(19) Lodi, L.; Tennyson, J.; Polyansky, O. L. A Global, High Accuracy Ab Initio Dipole Moment Surface for the Electronic Ground State of the Water Molecule. J. Chem. Phys. 2011, 135 (3), 034113. https://doi.org/10.1063/1.3604934.

(20) Tennyson, J. Accurate Variational Calculations for Line Lists to Model the Vibration-Rotation Spectra of Hot Astrophysical Atmospheres. Wiley Interdiscip. Rev. Comput. Mol. Sci. 2012, 2 (4), 698-715. https://doi.org/10.1002/wcms.94.

(21) Tennyson, J.; Yurchenko, S. N.; Al-Refaie, A. F.; Clark, V. H. J.; Chubb, K. L.; Conway, E. K.; Dewan, A.; Gorman, M. N.; Hill, C.; Lynas-Gray, A. E.; Mellor, T.; McKemmish, L. K.; Owens, A.; Polyansky, O. L.; Semenov, M.; Somogyi, W.; Tinetti, G.; Upadhyay, A.; Waldmann, I.; Wang, Y.; Wright, S.; Yurchenko, O. P. The 2020 Release of the ExoMol Database: Molecular Line Lists for Exoplanet and Other Hot Atmospheres. J. Quant. Spectrosc. Radiat. Transf. 2020, 255, 107228. https://doi.org/10.1016/j.jqsrt.2020.107228.

(22) Li, G.; Gordon, I. E.; Le Roy, R. J.; Hajigeorgiou, P. G.; Coxon, J. A.; Bernath, P. F.; Rothman, L. S. Reference Spectroscopic Data for Hydrogen Halides. Part I: Construction and Validation of the Ro-Vibrational Dipole Moment Functions. J. Quant. Spectrosc. Radiat. Transf. 2013, 121, 78-90. https://doi.org/10.1016/j.jqsrt.2013.02.005.

(23) Gastegger, M.; Behler, J.; Marquetand, P. Machine Learning Molecular Dynamics for the Simulation of Infrared Spectra. Chem. Sci. 2017, 8 (10), 6924-6935. https://doi.org/10.1039/c7sc02267k.

(24) Thomas, M.; Brehm, M.; Fligg, R.; Vöhringer, P.; Kirchner, B. Computing Vibrational Spectra from Ab Initio Molecular Dynamics. Phys. Chem. Chem. Phys. 2013, 15 (18), 6608-6622.

https://doi.org/10.1039/c3cp44302g.

(25) Gaigeot, M.-P. Theoretical Spectroscopy of Floppy Peptides at Room Temperature. A DFTMD Perspective: Gas and Aqueous Phase. Phys. Chem. Chem. Phys. 2010, 12 (14), 3336. https://doi.org/10.1039/b924048a.

(26) Fedorov, D. A.; Lykhin, A. O.; Varganov, S. A. Predicting Intersystem Crossing Rates with AIMS-DFT 
Molecular Dynamics. J. Phys. Chem. A 2018, 122 (13), 3480-3488.

https://doi.org/10.1021/acs.jpca.8b00883.

(27) Manni, G. L.; Carlson, R. K.; Luo, S.; Ma, D.; Olsen, J.; Truhlar, D. G.; Gagliardi, L. Multiconfiguration PairDensity Functional Theory. J. Chem. Theory Comput. 2014, 10 (9), 3669-3680.

https://doi.org/10.1021/ct500483t.

(28) Oakley, M. S.; Gagliardi, L.; Truhlar, D. G. Multiconfiguration Pair-Density Functional Theory for Transition Metal Silicide Bond Dissociation Energies, Bond Lengths, and State Orderings. Molecules 2021, 26 (10), 2881. https://doi.org/10.3390/molecules26102881.

(29) Lykhin, A. O.; Truhlar, D. G.; Gagliardi, L. Role of Triplet States in the Photodynamics of Aniline. J. Am. Chem. Soc. 2021, 143 (15), 5878-5889. https://doi.org/10.1021/jacs.1c00989.

(30) Ghosh, S.; Verma, P.; Cramer, C. J.; Gagliardi, L.; Truhlar, D. G. Combining Wave Function Methods with Density Functional Theory for Excited States. Chem. Rev. 2018, 118 (15), 7249-7292.

https://doi.org/10.1021/acs.chemrev.8b00193.

(31) Sand, A. M.; Kidder, K. M.; Truhlar, D. G.; Gagliardi, L. Calculation of Chemical Reaction Barrier Heights by Multiconfiguration Pair-Density Functional Theory with Correlated Participating Orbitals. J. Phys. Chem. A 2019, 123 (45), 9809-9817. https://doi.org/10.1021/acs.jpca.9b08134.

(32) Sharma, P.; Bernales, V.; Truhlar, D. G.; Gagliardi, L. Valence $\pi \pi^{*}$ Excitations in Benzene Studied by Multiconfiguration Pair-Density Functional Theory. J. Phys. Chem. Lett. 2019, 10 (1), 75-81. https://doi.org/10.1021/acs.jpclett.8b03277.

(33) Sharma, P.; Bernales, V.; Knecht, S.; Truhlar, D. G.; Gagliardi, L. Density Matrix Renormalization Group Pair-Density Functional Theory (DMRG-PDFT): Singlet-Triplet Gaps in Polyacenes and Polyacetylenes. Chem. Sci. 2019, 10 (6), 1716-1723. https://doi.org/10.1039/c8sc03569e.

(34) Roos, B. O.; Taylor, P. R.; Sigbahn, P. E. M. A Complete Active Space SCF Method (CASSCF) Using a Density Matrix Formulated Super-CI Approach. Chem. Phys. 1980, 48 (2), 157-173.

https://doi.org/10.1016/0301-0104(80)80045-0.

(35) Andersson, K.; Malmqvist, P.; Roos, B. O. Second-order Perturbation Theory with a Complete Active Space Self-consistent Field Reference Function. J. Chem. Phys. 1992, 96 (2), 1218-1226. https://doi.org/10.1063/1.462209.

(36) Knowles, P. J.; Werner, H. J. An Efficient Method for the Evaluation of Coupling Coefficients in Configuration Interaction Calculations. Chem. Phys. Lett. 1988, 145 (6), 514-522.

https://doi.org/10.1016/0009-2614(88)87412-8.

(37) Werner, H.; Knowles, P. J. An Efficient Internally Contracted Multiconfiguration-Reference Configuration Interaction Method. J. Chem. Phys. 1988, 89 (9), 5803-5814. https://doi.org/10.1063/1.455556.

(38) Langhoff, S. R.; Davidson, E. R. Configuration Interaction Calculations on the Nitrogen Molecule. Int. J. Quantum Chem. 1974, 8 (1), 61-72. https://doi.org/10.1002/qua.560080106.

(39) Yamaguchi, Y.; Frisch, M.; Gaw, J.; Schaefer, H. F.; Binkley, J. S. Analytic Evaluation and Basis Set Dependence of Intensities of Infrared Spectra. J. Chem. Phys. 1986, 84 (4), 2262-2278. https://doi.org/10.1063/1.450389.

(40) Pandharkar, R.; Hermes, M. R.; Truhlar, D. G.; Gagliardi, L. A New Mixing of Nonlocal Exchange and 
Nonlocal Correlation with Multiconfiguration Pair-Density Functional Theory. J. Phys. Chem. Lett. 2020, 11 (23), 10158-10163. https://doi.org/10.1021/acs.jpclett.0c02956.

(41) Sand, A. M.; Hoyer, C. E.; Sharkas, K.; Kidder, K. M.; Lindh, R.; Truhlar, D. G.; Gagliardi, L. Analytic Gradients for Complete Active Space Pair-Density Functional Theory. J. Chem. Theory Comput. 2018, 14 (1), 126-138. https://doi.org/10.1021/acs.jctc.7b00967.

(42) Scott, T. R.; Hermes, M. R.; Sand, A. M.; Oakley, M. S.; Truhlar, D. G.; Gagliardi, L. Analytic Gradients for State-Averaged Multiconfiguration Pair-Density Functional Theory. J. Chem. Phys. 2020, 153 (1), 014106. https://doi.org/10.1063/5.0007040.

(43) Scott, T. R.; Oakley, M. S.; Hermes, M. R.; Sand, A. M.; Lindh, R.; Truhlar, D. G.; Gagliardi, L. Analytic Gradients for Multiconfiguration Pair-Density Functional Theory with Density Fitting: Development and Application to Geometry Optimization in the Ground and Excited States. J. Chem. Phys. 2021, 154 (7), 074108. https://doi.org/10.1063/5.0039258.

(44) Sun, Q.; Zhang, X.; Banerjee, S.; Bao, P.; Barbry, M.; Blunt, N. S.; Bogdanov, N. A.; Booth, G. H.; Chen, J.; Cui, Z.-H.; Eriksen, J. J.; Gao, Y.; Guo, S.; Hermann, J.; Hermes, M. R.; Koh, K.; Koval, P.; Lehtola, S.; Li, Z.; Liu, J.; Mardirossian, N.; McClain, J. D.; Motta, M.; Mussard, B.; Pham, H. Q.; Pulkin, A.; Purwanto, W.; Robinson, P. J.; Ronca, E.; Sayfutyarova, E. R.; Scheurer, M.; Schurkus, H. F.; Smith, J. E. T.; Sun, C.; Sun, S.-N.; Upadhyay, S.; Wagner, L. K.; Wang, X.; White, A.; Whitfield, J. D.; Williamson, M. J.; Wouters, S.; Yang, J.; Yu, J. M.; Zhu, T.; Berkelbach, T. C.; Sharma, S.; Sokolov, A. Y.; Chan, G. K.-L. Recent Developments in the PySCF Program Package. J. Chem. Phys. 2020, 153 (2), 024109. https://doi.org/10.1063/5.0006074.

(45) Sun, Q.; Berkelbach, T. C.; Blunt, N. S.; Booth, G. H.; Guo, S.; Li, Z.; Liu, J.; McClain, J. D.; Sayfutyarova, E. R.; Sharma, S.; Wouters, S.; Chan, G. K. L. PySCF: The Python-Based Simulations of Chemistry Framework. Wiley Interdiscip. Rev. Comput. Mol. Sci. 2018, 8 (1). https://doi.org/10.1002/wcms.1340.

(46) Hermes, M. R. mrh https://github.com/MatthewRHermes/mrh. (accessed Sep 9, 2021).

(47) Sun, Q.; Yang, J.; Chan, G. K. L. A General Second Order Complete Active Space Self-Consistent-Field Solver for Large-Scale Systems. Chem. Phys. Lett. 2017, 683, 291-299.

https://doi.org/10.1016/j.cplett.2017.03.004.

(48) Bao, J. J.; Gagliardi, L.; Truhlar, D. G. Weak Interactions in Alkaline Earth Metal Dimers by Pair-Density Functional Theory. J. Phys. Chem. Lett. 2019, 10 (4), 799-805. https://doi.org/10.1021/acs.jpclett.8b03846.

(49) Carlson, R. K.; Truhlar, D. G.; Gagliardi, L. Multiconfiguration Pair-Density Functional Theory: A Fully Translated Gradient Approximation and Its Performance for Transition Metal Dimers and the Spectroscopy of $\mathrm{Re}_{2} \mathrm{Cl}_{8}^{2-}$. J. Chem. Theory Comput. 2015, 11 (9), 4077-4085. https://doi.org/10.1021/acs.jctc.5b00609.

(50) Sharkas, K.; Gagliardi, L.; Truhlar, D. G. Multiconfiguration Pair-Density Functional Theory and Complete Active Space Second Order Perturbation Theory. Bond Dissociation Energies of FeC, NiC, FeS, NiS, FeSe, and NiSe. J. Phys. Chem. A 2017, 121 (48), 9392-9400. https://doi.org/10.1021/acs.jpca.7b09779.

(51) Bao, J. L.; Odoh, S. O.; Gagliardi, L.; Truhlar, D. G. Predicting Bond Dissociation Energies of TransitionMetal Compounds by Multiconfiguration Pair-Density Functional Theory and Second-Order Perturbation Theory Based on Correlated Participating Orbitals and Separated Pairs. J. Chem. Theory Comput. 2017, 13 (2), 616-626. https://doi.org/10.1021/acs.jctc.6b01102. 
(52) Tishchenko, O.; Zheng, J.; Truhlar, D. G. Multireference Model Chemistries for Thermochemical Kinetics. J. Chem. Theory Comput. 2008, 4 (8), 1208-1219. https://doi.org/10.1021/ct800077r.

(53) Werner, H. J.; Kállay, M.; Gauss, J. The Barrier Height of the F+H2 Reaction Revisited: Coupled-Cluster and Multireference Configuration-Interaction Benchmark Calculations. J. Chem. Phys. 2008, 128 (3). https://doi.org/10.1063/1.2822905.

(54) Werner, H. J.; Knowles, P. J.; Manby, F. R.; Black, J. A.; Doll, K.; Heßelmann, A.; Kats, D.; Köhn, A.; Korona, T.; Kreplin, D. A.; Ma, Q.; Miller, T. F.; Mitrushchenkov, A.; Peterson, K. A.; Polyak, I.; Rauhut, G.; Sibaev, M. The Molpro Quantum Chemistry Package. J. Chem. Phys. 2020, 152 (14). https://doi.org/10.1063/5.0005081.

(55) Celani, P.; Werner, H. J. Analytical Energy Gradients for Internally Contracted Second-Order Multireference Perturbation Theory. J. Chem. Phys. 2003, 119 (10), 5044-5057. https://doi.org/10.1063/1.1597672.

(56) Zapata, J. C.; McKemmish, L. K. Computation of Dipole Moments: A Recommendation on the Choice of the Basis Set and the Level of Theory. J. Phys. Chem. A 2020, 124 (37), 7538-7548. https://doi.org/10.1021/acs.jpca.0c06736.

(57) Puzzarini, C. Isomerism of Cyanomethanimine: Accurate Structural, Energetic, and Spectroscopic Characterization. J. Phys. Chem. A 2015, 119 (47), 11614-11622. https://doi.org/10.1021/acs.jpca.5b09489.

(58) Tarroni, R.; Clouthier, D. J. Ab Initio Spectroscopy of the Aluminum Methylene (AICH2) Free Radical. J. Chem. Phys. 2020, 153 (1), 1-10. https://doi.org/10.1063/5.0010552.

(59) Papakondylis, A.; Mavridis, A. Electronic Structure and Bonding of the Fastidious Species CN2 and CP2: A First-Principles Study. J. Phys. Chem. A 2019, 123 (47), 10290-10302.

https://doi.org/10.1021/acs.jpca.9b09084.

(60) Takano, S.; Sugie, M.; Sugawara, K.; Takeo, H.; Matsumura, C.; Masuda, A.; Kuchitsu, K. Microwave Spectra, Dipole Moments, and Energy Difference of E and Z C-Cyanomethanimine (HCN Dimer). J. Mol. Spectrosc. 1990, 141 (1), 13-22. https://doi.org/10.1016/0022-2852(90)90273-S.

(61) Piecuch, P.; Kucharski, S. A.; Kowalski, K.; Musiał, M. Efficient Computer Implementation of the Renormalized Coupled-Cluster Methods : The R-CCSD [ T ], R-CCSD ( T ), CR-CCSD [ T ], and CR-CCSD ( T ) Approaches. 2002, 149, 71-96.

(62) Tennyson, J. Vibration-Rotation Transition Dipoles from First Principles. J. Mol. Spectrosc. 2014, $298,1-6$. https://doi.org/10.1016/j.jms.2014.01.012.

(63) Hait, D.; Head-Gordon, M. How Accurate Is Density Functional Theory at Predicting Dipole Moments? An Assessment Using a New Database of 200 Benchmark Values. J. Chem. Theory Comput. 2018, 14 (4), 1969-1981. https://doi.org/10.1021/acs.jctc.7b01252.

(64) Herman, R.; Wallis, R. F. Influence of Vibration-Rotation Interaction on Line Intensities in VibrationRotation Bands of Diatomic Molecules. J. Chem. Phys. 1955, 23 (4), 637-646. https://doi.org/10.1063/1.1742069.

(65) Tipping, R. H.; Herman, R. M. Line Intensities in HBr Vibration-Rotation Spectra. J. Mol. Spectrosc. 1970, 36 (3), 404-413. https://doi.org/10.1016/0022-2852(70)90217-1. 
(66) Li, G.; Gordon, I. E.; Bernath, P. F.; Rothman, L. S. Direct Fit of Experimental Ro-Vibrational Intensities to the Dipole Moment Function: Application to HCl. J. Quant. Spectrosc. Radiat. Transf. 2011, 112 (10), 1543-1550. https://doi.org/10.1016/j.jqsrt.2011.03.014.

(67) Medvedev, E. S.; Meshkov, V. V.; Stolyarov, A. V.; Ushakov, V. G.; Gordon, I. E. Impact of the DipoleMoment Representation on the Intensity of High Overtones. J. Mol. Spectrosc. 2016, 330, 36-42. https://doi.org/10.1016/j.jms.2016.06.013.

(68) Mann, D. E.; Thrush, B. A.; Lide, D. R.; Ball, J. J.; Acquista, N. Spectroscopy of Fluorine Flames. I. Hydrogen-Fluorine Flame and the Vibration-Rotation Emission Spectrum of HF. J. Chem. Phys. 1961, 34 (2), 420-431. https://doi.org/10.1063/1.1700967.

(69) Bass, S. M.; DeLeon, R. L.; Muenter, J. S. Stark, Zeeman, and Hyperfine Properties of V=0, V=1, and the Equilibrium Configuration of Hydrogen Fluoride. J. Chem. Phys. 1987, 86 (8), 4305-4312. https://doi.org/10.1063/1.451891.

(70) Muenter, J. S.; Klemperer, W. Hyperfine Structure Constants of HF and DF. J. Chem. Phys. 1970, 52 (12), 6033-6037. https://doi.org/10.1063/1.1672903.

(71) Gwaltney, S. R.; Head-Gordon, M. A Second-Order Perturbative Correction to the Coupled-Cluster Singles and Doubles Method: CCSD(2). J. Chem. Phys. 2001, 115 (5), 2014-2021. https://doi.org/10.1063/1.1383589.

(72) Coxon, J. A.; Hajigeorgiou, P. G. Improved Direct Potential Fit Analyses for the Ground Electronic States of the Hydrogen Halides: HF/DF/TF, $\mathrm{HCl} / \mathrm{DCl} / \mathrm{TCl}, \mathrm{HBr} / \mathrm{DBr} / \mathrm{TBr}$ and $\mathrm{HI} / \mathrm{DI} / \mathrm{TI}$. J. Quant. Spectrosc. Radiat. Transf. 2015, 151, 133-154. https://doi.org/10.1016/j.jqsrt.2014.08.028.

(73) Sileo, R. N.; Cool, T. A. Overtone Emission Spectroscopy of HF and DF: Vibrational Matrix Elements and Dipole Moment Function. J. Chem. Phys. 1976, 65 (1), 117-133. https://doi.org/10.1063/1.432808.

(74) Li, G.; Gordon, I. E.; Rothman, L. S.; Tan, Y.; Hu, S.-M.; Kassi, S.; Campargue, A.; Medvedev, E. S. Rovibrational Line Lists for Nine Isotopologues of the Co Molecule in the $X^{1} \Sigma^{+}$Ground Electronic State. Astrophys. J. Suppl. Ser. 2015, 216 (1), 15. https://doi.org/10.1088/0067-0049/216/1/15.

(75) Chackerian Jr., C.; Farrenq, R.; Guelachvili, G.; Rossetti, C.; Urban, W. Experimental Determination of the ${ }^{1} \Sigma^{+}$State Electric Dipole Moment Function of Carbon Monoxide up to a Large Internuclear Separation. Can. J. Phys. 1984, 62 (12), 1579-1585. https://doi.org/10.1139/p84-202.

(76) Coxon, J. A.; Hajigeorgiou, P. G. Direct Potential Fit Analysis of the $X^{1} \Sigma^{+}$Ground State of CO. J. Chem. Phys. 2004, 121 (7), 2992-3008. https://doi.org/10.1063/1.1768167.

(77) Muenter, J. S. Electric Dipole Moment of Carbon Monoxide. J. Mol. Spectrosc. 1975, 55 (1-3), 490-491. https://doi.org/10.1016/0022-2852(75)90287-8.

(78) Kiriyama, F.; Rao, B. S. Electric Dipole Moment Function of ${ }^{12} \mathrm{C}^{16} \mathrm{O}$. J. Quant. Spectrosc. Radiat. Transf. 2000, 65 (4), 673-679. https://doi.org/10.1016/S0022-4073(99)00138-7.

(79) Ogilvie, J. F.; Cheah, S. L.; Lee, Y. P.; Sauer, S. P. A. Infrared Spectra of CO in Absorption and Evaluation of Radial Functions for Potential Energy and Electric Dipolar Moment. Theor. Chem. Acc. 2002, 108 (2), 8597. https://doi.org/10.1007/s00214-002-0337-y.

(80) Buldakov, M. A.; Cherepanov, V. N. The Semiempirical Dipole Moment Functions of the Molecules HX (X $=\mathrm{F}, \mathrm{Cl}, \mathrm{Br}, \mathrm{I}, \mathrm{O}), \mathrm{CO}$ and NO. J. Phys. B At. Mol. Opt. Phys. 2004, 37 (19), 3973-3986. 
https://doi.org/10.1088/0953-4075/37/19/015.

(81) Chung, C.-Y.; Ogilvie, J. F.; Lee, Y. Detection of Vibration-Rotational Band 5-0 of ${ }^{12} \mathrm{C}^{16} \mathrm{O} X{ }^{1} \Sigma^{+}$with Cavity Ringdown Absorption near 0.96 m. J. Phys. Chem. A 2005, 109 (35), 7854-7858.

https://doi.org/10.1021/jp052035x.

(82) Chen, H.-J.; Wu, J.; Liu, H.; Cheng, X.-L. Electric Dipole Moment Function and Line Intensities for the Ground State of Carbon Monxide. Chinese Phys. B 2015, 24 (8), 083102. https://doi.org/10.1088/16741056/24/8/083102.

(83) Langhoff, S. R.; Bauschlicher, C. W. Global Dipole Moment Function for the $X^{1} \Sigma^{+}$Ground State of CO. J. Chem. Phys. 1995, 102 (13), 5220-5225. https://doi.org/10.1063/1.469247.

(84) Qin, Z.; Zhao, J. M.; Liu, L. H. Radiative Transition Probabilities for the Main Diatomic Electronic Systems of $\mathrm{N}_{2}, \mathrm{~N}_{2}{ }^{+}, \mathrm{NO}, \mathrm{O}_{2}, \mathrm{CO}, \mathrm{CO}^{+}, \mathrm{CN}, \mathrm{C}_{2}$ and $\mathrm{H}_{2}$ Produced in Plasma of Atmospheric Entry. J. Quant. Spectrosc. Radiat. Transf. 2017, 202, 286-301. https://doi.org/10.1016/j.jqsrt.2017.08.010.

(85) Liu, Y.; Guo, Y.; Lin, J.; Huang, G.; Duan, C.; Li, F. Measurement of the Electric Dipole Moment of NO (X ${ }^{2} \Pi$ $v=0,1$ ) by Mid-Infrared Laser Magnetic Resonance Spectroscopy. Mol. Phys. 2001, 99 (17), 1457-1461. https://doi.org/10.1080/00268970110059642.

(86) Wong, A.; Yurchenko, S. N.; Bernath, P.; Müller, H. S. P.; McConkey, S.; Tennyson, J. ExoMol Line List XXI. Nitric Oxide (NO). Mon. Not. R. Astron. Soc. 2017, 470 (1), 882-897. https://doi.org/10.1093/mnras/stx1211.

(87) Bood, J.; Mcllroy, A.; Osborn, D. L. Measurement of the Sixth Overtone Band of Nitric Oxide, and Its Dipole Moment Function, Using Cavity-Enhanced Frequency Modulation Spectroscopy. J. Chem. Phys. 2006, 124 (8), 084311. https://doi.org/10.1063/1.2170090.

(88) Launila, O.; Jonsson, J. Spectroscopy of AIO: Rotational Analysis of the $A^{2} \Pi_{i}-X^{2} \Sigma^{+}$Transition in the $2-\mu m$ Region. J. Mol. Spectrosc. 1994, 168 (1), 1-38. https://doi.org/10.1006/jmsp.1994.1257.

(89) Bai, X.; Steimle, T. C. The Stark Effect, Zeeman Effect, and Transition Dipole Moments for the B ${ }^{2} \Sigma^{+}-X^{2} \Sigma^{+}$ Band of Aluminum Monoxide, AIO. Astrophys. J. 2020, 889 (2), 147. https://doi.org/10.3847/15384357/ab6327.

(90) Breier, A. A.; Waßmuth, B.; Büchling, T.; Fuchs, G. W.; Gauss, J.; Giesen, T. F. A Mass-Independent Expanded Dunham Analysis of Aluminum Monoxide and Aluminum Monosulfide. J. Mol. Spectrosc. 2018, 350, 43-50. https://doi.org/10.1016/j.jms.2018.06.001.

(91) Lengsfield, B. H.; Liu, B. Ab Initio Dipole Moment Functions for the $X^{2} \Sigma^{+}$and B ${ }^{2} \Sigma^{+}$States of AlO. J. Chem. Phys. 1982, 77 (12), 6083-6089. https://doi.org/10.1063/1.443852.

(92) Patrascu, A. T.; Yurchenko, S. N.; Tennyson, J. ExoMol Molecular Line Lists - IX. The Spectrum of AIO. Mon. Not. R. Astron. Soc. 2015, 449 (4), 3613-3619. https://doi.org/10.1093/mnras/stv507.

(93) Steimle, T. C. Permanent Electric Dipole Moments of Metal Containing Molecules. Int. Rev. Phys. Chem. 2000, 19 (3), 455-477. https://doi.org/10.1080/01442350050034199.

(94) Liu, X.; Meijer, G.; Pérez-Ríos, J. A Data-Driven Approach to Determine Dipole Moments of Diatomic Molecules. Phys. Chem. Chem. Phys. 2020, 22 (42), 24191-24200. https://doi.org/10.1039/d0cp03810e.

(95) Simard, B.; Vasseur, M.; Hackett, P. A. The Permanent Dipole Moment of ScF. Chem. Phys. Lett. 1991, 176 (3-4), 303-308. https://doi.org/10.1016/0009-2614(91)90034-7. 
(96) Le, A.; Steimle, T. C. Optical Stark Spectroscopy of the $D^{1} \Pi-X^{1} \Sigma^{+}(0,0)$ Band of Scandium Monohydride. J. Phys. Chem. A 2011, 115 (34), 9370-9376. https://doi.org/10.1021/jp110838j.

(97) Zhuang, X.; Steimle, T. C. The Permanent Electric Dipole Moment of Vanadium Monosulfide. J. Chem. Phys. 2010, 132 (23), 234304. https://doi.org/10.1063/1.3454722.

(98) Steimle, T. C.; Shirley, J. E. The Stark Effect in the B ${ }^{3} \Pi-X^{3} \Delta$ Band System of TiO. J. Chem. Phys. 1989, 91 (12), 8000-8002. https://doi.org/10.1063/1.457222.

(99) Steimle, T. C.; Virgo, W. The Permanent Electric Dipole Moments of the $X^{3} \Delta, E{ }^{3} \Pi, A^{3} \Phi$ and $B{ }^{3} \Pi$ States of Titanium Monoxide, TiO. Chem. Phys. Lett. 2003, 381 (1-2), 30-36. https://doi.org/10.1016/j.cplett.2003.09.102.

(100) Chen, J.; Steimle, T. C.; Merer, A. J. The Permanent Electric Dipole Moment of Chromium Monodeuteride, CrD. J. Chem. Phys. 2007, 127 (20), 204307. https://doi.org/10.1063/1.2800003.

(101) Kłos, J.; Hapka, M.; Chałasiński, G.; Halvick, P.; Stoecklin, T. Theoretical Study of the Buffer-Gas Cooling and Trapping of $\mathrm{CrH}\left(\mathrm{X}^{6} \Sigma^{+}\right)$by ${ }^{3} \mathrm{He}$ Atoms. J. Chem. Phys. 2016, 145 (21), 214305. https://doi.org/10.1063/1.4968529.

(102) Miliordos, E.; Mavridis, A. Electronic Structure and Bonding of the Early 3d-Transition Metal Diatomic Oxides and Their lons: $\mathrm{ScO}^{0, \pm}, \mathrm{TiO}^{0, \pm}, \mathrm{CrO}^{0, \pm}$, and $\mathrm{MnO}^{0, \pm}$. J. Phys. Chem. A 2010, 114 (33), 8536-8572. https://doi.org/10.1021/jp910218u.

(103) Shirley, J.; Scurlock, C.; Steimle, T. Molecular-beam Optical Stark Spectroscopy of ScO. J. Chem. Phys. 1990, 93 (3), 1568-1575. https://doi.org/10.1063/1.459135.

(104) Wang, H.; Zhuang, X.; Steimle, T. C. The Permanent Electric Dipole Moments of Cobalt Monofluoride, CoF, and Monohydride, CoH. J. Chem. Phys. 2009, 131 (11), 114315. https://doi.org/10.1063/1.3226672.

(105) Sakellaris, C. N.; Mavridis, A. First Principles Study of Cobalt Hydride, $\mathrm{CoH}$, and Its lons $\mathrm{CoH}^{+}$and $\mathrm{CoH}^{-}$. J. Chem. Phys. 2012, 137 (3), 034309. https://doi.org/10.1063/1.4734595.

(106) de Moraes, M. M. F.; Aoto, Y. A. Reference Spaces for Multireference Coupled-Cluster Theory: The Challenge of the CoH Molecule. Theor. Chem. Acc. 2020, 139 (4), 71. https://doi.org/10.1007/s00214020-2584-1.

(107) Aoto, Y. A.; de Lima Batista, A. P.; Köhn, A.; de Oliveira-Filho, A. G. S. How To Arrive at Accurate Benchmark Values for Transition Metal Compounds: Computation or Experiment? J. Chem. Theory Comput. 2017, 13 (11), 5291-5316. https://doi.org/10.1021/acs.jctc.7b00688.

(108) Fujitake, M.; Toba, A.; Mori, M.; Miyazawa, F.; Ohashi, N.; Aiuchi, K.; Shibuya, K. Near-Infrared Diode Laser Spectroscopy of $\mathrm{FeC}$ in the $0.8-\mu \mathrm{m}$ Region: A Simultaneous Analysis of the $\mathrm{X}^{3} \Delta_{\mathrm{i}}$ and [3.8 $]^{1} \Delta$ States. J. Mol. Spectrosc. 2001, 208 (2), 253-270. https://doi.org/10.1006/jmsp.2001.8399.

(109) Beaton, S. P.; Evenson, K. M.; Brown, J. M. Rotational Spectroscopy of the CoH Radical in Its Ground ${ }^{3} \Phi$ State by Far-Infrared Laser Magnetic Resonance: Determination of Molecular Parameters. J. Mol. Spectrosc. 1994, 164 (2), 395-415. https://doi.org/10.1006/jmsp.1994.1084.

(110) Tomonari, M.; Okuda, R.; Nagashima, U.; Tanaka, K.; Hirano, T. Ab Initio Calculation of the Electronic Structures of the ${ }^{3} \Phi$ Ground and ${ }^{5} \Phi$ Excited States of CoH. J. Chem. Phys. 2007, 126 (14), 144307. https://doi.org/10.1063/1.2711193.

(111) Deyonker, N. J.; Allen, W. D. Taming the Low-Lying Electronic States of FeH. J. Chem. Phys. 2012, 137 
(23). https://doi.org/10.1063/1.4767771.

(112) Cazzoli, G.; Puzzarini, C.; Baldacci, A.; Baldan, A. Determination of the Molecular Dipole Moment of Bromofluoromethane: Microwave Stark Spectra and Ab Initio Calculations. J. Mol. Spectrosc. 2007, 241 (1), 112-115. https://doi.org/10.1016/j.jms.2006.11.004.

(113) Paulson, L. O.; Kaminský, J.; Anderson, D. T.; Bouř, P.; Kubelka, J. Theoretical Study of Vibrationally Averaged Dipole Moments for the Ground and Excited $\mathrm{C}=0$ Stretching States of Trans-Formic Acid. J. Chem. Theory Comput. 2010, 6 (3), 817-827. https://doi.org/10.1021/ct900608t.

(114) Dai, D. G.; Balasubramanian, K. Spectroscopic Properties and Potential Energy Curves for 21 Electronic States of CrH. J. Mol. Spectrosc. 1993, 161 (2), 455-465. https://doi.org/10.1006/jmsp.1993.1251.

(115) Ghigo, G.; Roos, B. O.; Stancil, P. C.; Weck, P. F. A Theoretical Study of the Excited States of CrH: Potential Energies, Transition Moments, and Lifetimes. J. Chem. Phys. 2004, 121 (17), 8194-8200. https://doi.org/10.1063/1.1794631.

(116) Chen, Y. M.; Clemmer, D. E.; Armentrout, P. B. Gas-Phase Thermochemistry of VH and CrH. J. Chem. Phys. 1993, 98 (6), 4929-4936. https://doi.org/10.1063/1.464948.

(117) Handy, N. C.; Cohen, A. J. Left-Right Correlation Energy. Mol. Phys. 2001, 99 (5), 403-412. https://doi.org/10.1080/00268970010018431.

(118) King, D. S.; Truhlar, D. G.; Gagliardi, L. Machine-Learned Energy Functionals for Multiconfigurational Wave Functions. J. Phys. Chem. Lett. 2021, No. 1, 7761-7767.

https://doi.org/10.1021/acs.jpclett.1c02042.

For Table of Contents Only

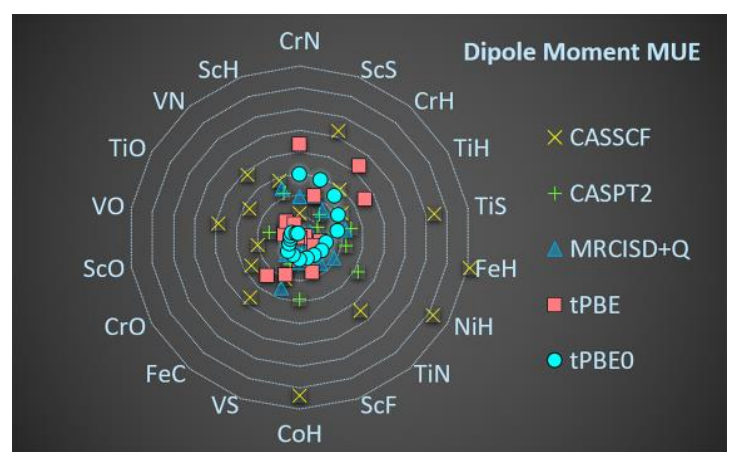

